\title{
THE QUEST FOR STRONG DUALITIES
}

\author{
DAVID M. CLARK and BRIAN A. DAVEY
}

(Received 24 November 1992; Revised 28 June 1993)

Communicated by R. Lidl

\begin{abstract}
We give a revised and updated exposition of the theory of full dualities initiated by Clark, Davey, Krauss and Werner, introducing the (stronger) notion of a strong duality. All known full dualities turn out to be strong. A series of theorems which provide necessary and sufficient conditions for a strong duality to exist is proved. All full dualities in the literature can be obtained from these results and many new strong dualities can be derived. In particular, we show that within congruence distributive varieties every duality can be upgraded to a strong duality. Amongst the new strong dualities are the dualities of Davey, Priestley and Werner for the varieties of pseudocomplemented distributive lattices which are either strong as they stand or can easily be made strong by the addition of partial operations to the dual structures.
\end{abstract}

1991 Mathematics subject classification (Amer. Math. Soc.): 08C05, 08C15, $18 \mathrm{~A} 40$.

Keywords and phrases: duality theory, full duality, strong duality, congruence distributivity, near unanimity.

A topological duality provides us with a uniform way to represent each algebra in the quasi-variety $\mathscr{A}=\mathbb{N S P} \underline{\mathbf{M}}$ generated by a finite algebra $\underline{\mathbf{M}}$ as the algebra of all (continuous) morphisms over its associated dual space in some category $\mathscr{X}$ of structured Boolean spaces. This approach originated with Stone's representation theorem for Boolean algebras [23] and Birkhoff's representation for finite distributive lattices [2]. In the late 1960s and early 1970s it gained considerable impetus from the very useful dualities of Priestley for all distributive lattices [22] and of Hofmann, Mislove and Stralka for semilattices [19].

A number of additional examples culminated in a general theory of topological dualities for finitely generated quasi-varieties which appeared in Davey and Werner [16]. Their approach is to impose on the carrier $M$ of $\underline{\mathbf{M}}$ the discrete topology together with a collection of carefully chosen operations, partial operations and relations to form

(C) 1995 Australian Mathematical Society 0263-6115/95 \$A2.00+0.00

Research supported by a 1992 ARC Grant (Davey) 
a dual structure $\mathbf{M}$ as generator of a dual category $\mathscr{X}$. If this is done in a special but very natural way, then the homsets $E(\mathbf{X})=\mathscr{X}(\mathbf{X}, \mathbf{M})$, where $\mathbf{X} \in \mathscr{X}$, and $D(\mathbf{A})=\mathscr{A}(\mathbf{A}, \underline{\mathbf{M}})$, where $\mathbf{A} \in \mathscr{A}$, will be (closed) substructures of the powers $\underline{\mathbf{M}}^{X}$ and $\mathbf{M}^{A}$ respectively, and $E: \mathscr{X} \rightarrow \mathscr{A}$ and $D: \mathscr{A} \rightarrow \mathscr{X}$ will be a pair of adjoint contravariant functors such that each $\mathbf{A} \in \mathscr{A}$ is concretely represented as $\mathbf{A} \cong E D(\mathbf{A})$. In this case we say that $\mathbf{M}$ yields a duality on $\mathscr{A}$.

The association of algebras and homomorphisms in $\mathscr{A}$ with topological structures and morphisms in $\mathscr{X}$ provides not only a concrete representation of the algebras, but also preserves much of the structure of these categories as well. This fact led Davey and Werner [16] and, more extensively, Clark and Krauss [5] to investigate choices of $\mathscr{X}$ for which the association would be a dual equivalence between the categories $\mathscr{A}$ and $\mathscr{X}$. When this happens we say that $\mathbf{M}$ yields a full duality on $\mathscr{A}$.

A review of the literature shows that a number of additional features may be present under a full duality which do not follow from category dual equivalence.
[A] Embeddings in $\mathscr{X}$ may be associated with surjections in $\mathscr{A}$.
[B] $\mathbf{M}$ may be injective in $\mathscr{X}$.
[C] Embeddings in $\mathscr{A}$ may be associated with surjections in $\mathscr{X}$.

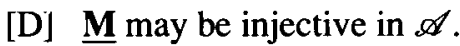

In the presence of full duality it will not be hard to show that $[\mathrm{A}]$ and $[\mathrm{B}]$ are equivalent and are implied by $[C]$ and $[D]$, which are also equivalent. This study is built around the observation that all existing proofs of full duality actually establish a condition which is stronger, more natural and more useful than full duality. We will say that $\mathbf{M}$ yields a strong duality when this condition is met. Serendipitously, strong duality always gives us $[\mathrm{A}]$ and $[\mathrm{B}]$ as well. Because strong duality provides our only apparent access to full duality and is required for almost all interesting full duality theorems and applications, we have made it the central focus of this study. We will show in a later paper that conditions [C] and [D] hold exactly when the strong duality can be obtained without the use of partial operations in the dual category.

Building on the work of Davey and Werner [16] and Clark and Krauss [5], we will develop a number of methods for modifying a choice of $\mathbf{M}$, which is already known to yield a duality, to obtain one which yields a strong duality by adding operations as well as partial operations to its structure. In particular, we will see just why partial operations are in general necessary to do this. While it is not known if every duality can be upgraded to a full duality or to a strong duality, the efficacy of the methods presented here is evidenced by the fact that they can now be successfully applied to upgrade every known duality to a strong duality by adding finitely many (partial) operations to $\mathbf{M}$.

One recurrent theme in our development will be the schizophrenic nature of the various personalities we encounter in the categories $\mathscr{A}$ and $\mathscr{X}$. Another will be the 
frequent identification of finitary versions of the conditions that interest us, with the ultimate hope of showing that the finitary version is sufficient to establish the condition in general.

Recent literature has shown that full dualities (which were actually strong dualities) provide a very detailed understanding of the quasi-variety $\mathscr{A}$. In these cases many questions that arise in $\mathscr{A}$ can be translated, via a strong duality, into equivalent (yet often much easier) problems in the dual category $\mathscr{X}$. Such translations depend on our ability to formulate questions fully in terms of algebras and their homomorphisms, although they are not always given in this form. For example, the compactness theorem for propositional logic translates into the assertion that the dual space of the Lindenbaum algebra is compact (Stone [23]). Strong dualities were used by Cornish and Fowler to characterize free de Morgan algebras and coproducts of de Morgan algebras in [7]. Adams and Clark [1] use them to reconstruct algebras in certain quasi-primal varieties from their endomorphism monoids. They are used by Davey, Quackenbush and Schweigert [15] to determine which order-primal algebras generate congruence distributive varieties. Clark uses them to provide a general technique for identifying and axiomatizing algebraically and existentially closed structures in $\mathscr{A}$, and this is applied to Stone algebras and double Stone algebras in Clark [3] and to the variety $\mathscr{B}_{2}$ of pseudocomplemented distributive lattices in Clark and Schmid [6].

In Section 1 we review the necessary background from Davey and Werner [16]. This material can be found in an updated though outline form in Davey [11] and will soon appear in the full exposition of Clark and Davey [4]. Section 2 constitutes a revised, simplified and updated formulation of the material in Clark and Krauss [5] on full dualities. In Section 3 we show how the notion of strong duality arises, prove that it is equivalent to full duality plus the injectivity of $\mathbf{M}$ in $\mathscr{X}$, and we prove three general theorems telling how it can be obtained. These efforts culminate in the next section where we prove the Two-for-One Strong Duality Theorem and the NU-StrongDuality Theorem, each of which gives finitary conditions for a strong duality to exist. Some readers may wish to familiarize themselves with the definitions and then go directly to these theorems to obtain a strong duality for their favorite quasivarieties. We leave it to the reader to check that these theorems do convert every existing full duality into a strong duality, or to see this fact checked directly in the upcoming text by the authors [4]. (Added in proof. Recently, R. W. Quackenbush and the second author have established strong dualities (for certain varieties of groups and quasigroups), which do not follow directly from the results of this paper, thereby opening up the possibility of theorems more general than those proved here.) 


\section{Dual adjunctions and duality}

We begin by laying out the few category theoretic concepts necessary for our work. Suppose that $\mathscr{A}$ and $\mathscr{X}$ are categories, $D: \mathscr{A} \rightarrow \mathscr{X}$ and $E: \mathscr{X} \rightarrow \mathscr{A}$ are contravariant functors and for each $\mathbf{A} \in \mathscr{A}$ and $\mathbf{X} \in \mathscr{X}$ there are morphisms

$$
e_{\mathbf{A}}: \mathbf{A} \rightarrow E D(\mathbf{A}) \quad \text { and } \quad \varepsilon_{\mathbf{X}}: \mathbf{X} \rightarrow D E(\mathbf{X}) .
$$

We say that $\langle D, E, e, \varepsilon\rangle$ is a dual adjunction between $\mathscr{A}$ and $\mathscr{X}$, that $D$ and $E$ are dually adjoint, that $E$ is a dual adjoint to $D$, and that $D$ is a dual adjoint to $E$ if the following conditions hold:

(a) for $u: \mathbf{A} \rightarrow \mathbf{B}$ in $\mathscr{A}$ and $\varphi: \mathbf{X} \rightarrow \mathbf{Y}$ in $\mathscr{X}$, the diagrams in Figure 1 commute.

(b) for $\mathbf{A} \in \mathscr{A}$ and $\mathbf{X} \in \mathscr{X}$ there is a bijection between $\mathscr{A}(\mathbf{A}, E(\mathbf{X}))$ and $\mathscr{X}(\mathbf{X}, D(\mathbf{A}))$ associating $u$ and $\varphi$ as given in the commuting diagrams of Figure 2.
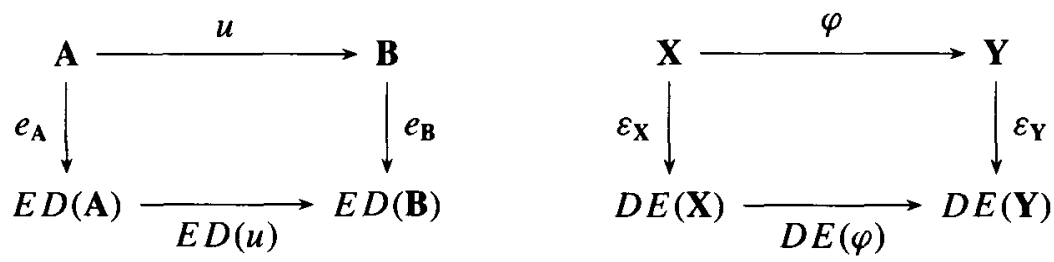

FIGURE 1
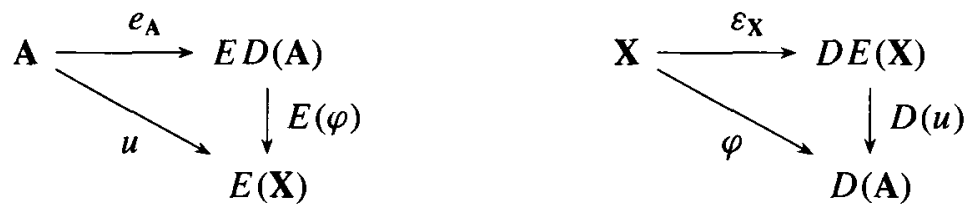

FIGURE 2

General duality theory grows out of a simple mechanism of Davey and Wener [16] for generating a profusion of dual adjunctions for the quasi-variety $\mathscr{A}=\square \mathbb{S P M}$ generated by any finite algebra $\underline{\mathbf{M}}$. To present their method we must first describe the objects of the dual category $\mathscr{X}$. They will be topological structures $\mathbf{X}=\left\langle X ; G^{X}, H^{X}, R^{X}, \mathscr{T}^{X}\right\rangle$ where $\mathscr{T}^{X}$ is a topology on $X$ and $G^{X}$ is a set of finitary total operations, $H^{X}$ is a set of finitary partial operations and $R^{X}$ is a set of finitary relations on $X$.

Since the usual constructions are not fully standardized when partial operations are present, we will review them here. Let $G, H$ and $R$ be fixed sets of finitary operation symbols, partial operation symbols and relation symbols, each carrying its own finite 
arity. If $\mathbf{Y}=\left\langle Y ; G^{Y}, H^{Y}, R^{Y}, \mathscr{T}^{Y}\right\rangle$ is a topological structure of the same type as $\mathbf{X}$, we say that $\mathbf{Y}$ is a substructure of $\mathbf{X}$, written $\mathbf{Y} \leq \mathbf{X}$, provided that

(a) for each $n$-ary $h \in G \cup H$, the domain $\operatorname{dom}\left(h^{Y}\right)$ of $h^{Y}$ is $\operatorname{dom}\left(h^{X}\right) \cap Y^{n}$, and $h^{Y}$ agrees with $h^{X}$ on this set,

(b) for each $n$-ary $r \in R$, we have $r^{Y}=r^{X} \cap Y^{n}$, and

(c) $\mathscr{T}^{Y}$ is the relative topology obtained from $\mathscr{T}^{X}$.

Products are defined over nonempty index sets in the usual manner using pointwise (total) operations and relations and the product topology, and partial operations are defined (pointwise) whenever they are defined at each point.

A continuous map $\varphi: X \rightarrow Y$ is a morphism, written $\varphi: \mathbf{X} \rightarrow \mathbf{Y}$, if for each $n$-ary $h \in G \cup H$ and each $\left(x_{1}, \ldots, x_{n}\right) \in \operatorname{dom}\left(h^{X}\right)$, we have $\left(\varphi x_{1}, \ldots, \varphi x_{n}\right) \in \operatorname{dom}\left(h^{Y}\right)$ and

$$
\varphi h^{X}\left(x_{1}, \ldots, x_{n}\right)=h^{Y}\left(\varphi x_{1}, \ldots, \varphi x_{n}\right)
$$

and for each $n$-ary $r \in R$ and each $\left(x_{1}, \ldots, x_{n}\right) \in r^{X}$ we have $\left(\varphi x_{1}, \ldots, \varphi x_{n}\right) \in r^{Y}$. A morphism $\varphi: \mathbf{X} \rightarrow \mathbf{Y}$ is an embedding if it is one-to-one, $\varphi(X)$ determines a substructure $\varphi(\mathbf{X})$ of $\mathbf{Y}$ and $\varphi^{-1}: \varphi(\mathbf{X}) \rightarrow \mathbf{X}$ is a morphism. A surjective embedding is an isomorphism.

For a class $\mathscr{Y}$ of similar topological structures, $\mathbb{\nabla}, \mathbb{S}_{c} \mathscr{Y}, \mathbb{P} \mathscr{Y}$ denote, respectively, the classes of isomorphic copies of members of $\mathscr{Y}$, topologically closed substructures of members of $\mathscr{Y}$, and products (over non-empty index sets) of members of $\mathscr{Y}$.

We will normally suppress the superscripts on the (partial) operation and relation symbols when the associated structure is clear from the context.

Given a finite algebra $\underline{\mathbf{M}}$, we seek a structure $\mathbf{M}=\langle M ; G, H, R, \mathscr{T}\rangle$ which shares the carrier $M$ of $\underline{\mathbf{M}}$ and has the discrete topology $\mathscr{T}$ such that there is a dual adjunction $\langle D, E, e, \varepsilon\rangle$ between the quasi-variety $\mathscr{A}=\mathbb{\Lambda S P} \underline{\mathbf{M}}$ and the topological category $\mathscr{X}=\| \mathbb{S}_{c} \mathbb{P} \mathbf{M}$. Our first lemma gives a useful characterization of any such category $\mathscr{X}$.

1.1 SEPARATION LEMMA. (Clark and Krauss [5, 1.3]) Let $\mathbf{Y}$ be a compact structure of the same type as the finite discretely topologized structure $\mathbf{M}$. Then $\mathbf{Y} \in \mathscr{X}=$ $\square S_{c} \mathbb{P M}$ if and only if the following conditions hold:

(a) for each $x, y \in Y$ where $x \neq y$, there is $a \varphi: \mathbf{Y} \rightarrow \mathbf{M}$ such that $\varphi(x) \neq \varphi(y)$,

(b) for each $n$-ary $h \in H$ and $y \in Y^{n} \backslash \operatorname{dom}\left(h^{Y}\right)$, there is a $\varphi: \mathbf{Y} \rightarrow \mathbf{M}$ such that $\varphi(y) \notin \operatorname{dom}\left(h^{M}\right)$,

(c) for each $n$-ary $r \in R$ and $y \in Y^{n} \backslash r^{Y}$, there is $a \varphi: \mathbf{Y} \rightarrow \mathbf{M}$ such that $\varphi(y) \notin r^{M}$.

We say that an operation, a partial operation or a relation on $\mathbf{M}$ is algebraic over $\underline{\mathbf{M}}$ if it is a subalgebra of a power of $\underline{\mathbf{M}}$. Notice that an $n$-ary (partial) operation $h \subseteq \bar{M}^{n+1}$ is algebraic over $\underline{\mathbf{M}}$ if and only if it is a homomorphism from a subalgebra of $\underline{\mathbf{M}}^{n}$ into 
$\underline{\mathbf{M}}$. We say that $\mathbf{M}$ is algebraic over $\underline{\mathbf{M}}$ if each relation and each (partial) operation of $\mathbf{M}$ is a subalgebra of a power of $\underline{\mathbf{M}}$.

1.2 LEMMA. (Davey and Werner [16,1.1]) For a finite algebra $\underline{\mathbf{M}}$ and a discrete topological structure $\mathbf{M}$ having the same carrier $M$ as $\underline{\mathbf{M}}$, conditions (b), (c) and (d) below are equivalent and imply condition (a):

(a) for each $\mathbf{A} \in \mathscr{A}$, the homset $\mathscr{A}(\mathbf{A}, \underline{\mathbf{M}})$ determines a closed substructure of $\mathbf{M}^{A}$;

(b) for each $\mathbf{X} \in \mathscr{X}$, the homset $\mathscr{X}(\mathbf{X}, \mathbf{M})$ determines a subalgebra of $\underline{\mathbf{M}}^{X}$;

(c) each operation (and therefore each term function) of $\underline{\mathbf{M}}$ is a morphism from a power of $\mathbf{M}$ into $\mathbf{M}$ and each nullary operation (constant) of $\underline{\mathbf{M} \text { determines a }}$ substructure of $\mathbf{M}$ all of whose relations and partial operations are nonempty;

(d) $\mathbf{M}$ is algebraic over $\underline{\mathbf{M}}$.

If $\mathbf{M}$ is algebraic over $\underline{\mathbf{M}}$, we can define $D: \mathscr{A} \rightarrow \mathscr{X}$ and $E: \mathscr{X} \rightarrow \mathscr{A}$ where, for $\mathbf{A}, \mathbf{B} \in \mathscr{A}$ and $u: \mathbf{A} \rightarrow \mathbf{B}$

$$
D(\mathbf{A})=\mathscr{A}(\mathbf{A}, \underline{\mathbf{M}}) \leq \mathbf{M}^{A}
$$

and $D(u): D(\mathbf{B}) \rightarrow D(\mathbf{A})$ is given by $D(u)(x)=x \circ u$ and, for $\mathbf{X}, \mathbf{Y} \in \mathscr{X}$ and $\varphi: \mathbf{X} \rightarrow \mathbf{Y}$,

$$
E(\mathbf{X})=\mathscr{X}(\mathbf{X}, \mathbf{M}) \leq \underline{\mathbf{M}}^{X}
$$

and $E(\varphi): E(\mathbf{Y}) \rightarrow E(\mathbf{X})$ is given by $E(\varphi)(\alpha)=\alpha \circ \varphi$. Moreover, for each $\mathbf{A} \in \mathscr{A}$ and each $\mathbf{X} \in \mathscr{X}$ we define the evaluation maps

$$
e_{\mathrm{A}}: \mathbf{A} \rightarrow E D(\mathbf{A}) \quad \text { by } \quad e_{\mathrm{A}}(a)(x)=x(a)
$$

and

$$
\varepsilon_{\mathbf{X}}: \mathbf{X} \rightarrow D E(\mathbf{X}) \quad \text { by } \quad \varepsilon_{\mathbf{X}}(x)(\alpha)=\alpha(x)
$$

(See Figure 3.)

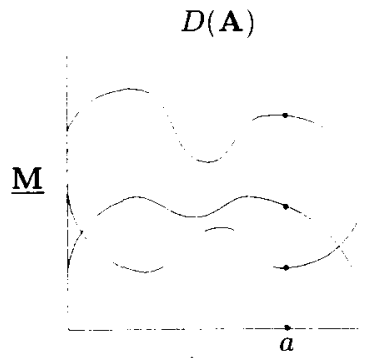

A

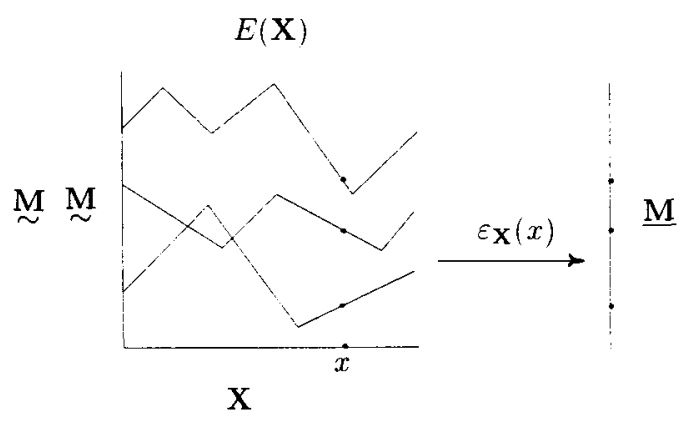

FIGURE 3 
The next theorem, which is straightforward to verify, shows that in this context a large part of the construction we seek is present as soon as $\mathbf{M}$ is algebraic over $\underline{\mathbf{M}}$.

1.3 DuAl AdJunction TheOREM. (Davey and Werner [16, 1.5]) If $\mathbf{M}$ is algebraic over $\underline{\mathbf{M}}$, then $\langle D, E, e, \varepsilon\rangle$ is a dual adjunction between $\mathscr{A}$ and $\mathscr{X}$ such that, for any $u: \mathbf{A} \rightarrow \mathbf{B}$ in $\mathscr{A}$ and $\varphi: \mathbf{X} \rightarrow \mathbf{Y}$ in $\mathscr{X}$, the following additional properties hold:

(a) the evaluation maps $e_{\mathrm{A}}$ and $\varepsilon_{\mathrm{X}}$ are embeddings,

(b) D and E are faithful,

(c) if $u$ is a surjection, then $D(u)$ is an embedding,

(d) if $D(u)$ is a surjection, then $u$ is an embedding,

(e) if $\varphi$ is a surjection, then $E(\varphi)$ is an embedding,

(f) if $E(\varphi)$ is a surjection, then $\varphi$ is an embedding.

The remainder of this paper is devoted exclusively to the study of the setting described in the Dual Adjunction Theorem. In particular, there will never be occasion to refer to a choice of a structure $\mathbf{M}$ which is not algebraic over $\underline{\mathbf{M}}$. We therefore assume without further mention that all operations, partial operations and relations on $\mathbf{M}$ are algebraic over $\underline{\mathbf{M}}$.

A dual adjunction reflects the structure of each of the categories $\mathscr{A}$ and $\mathscr{X}$ in the other. From the algebraic point of view, our first aim must be to represent each algebra $\mathbf{A} \in \mathscr{A}$ as the algebra $E(\mathbf{X})=\mathscr{X}(\mathbf{X}, \mathbf{M})$ of all structure preserving maps (that is, morphisms) from $\mathbf{X}$ into $\mathbf{M}$. A dual adjunction provides us with a very natural choice, namely $\mathbf{X}=D(\mathbf{A})$, for the representing space as well as a very natural choice, $e_{\mathbf{A}}: \mathbf{A} \rightarrow E D(\mathbf{A})$, for the required isomorphism. Consequently we say that $\mathbf{M}$ yields a duality on $\mathscr{A}$ if it is algebraic over $\underline{\mathbf{M}}$ (and therefore yields a dual adjunction) and for each $\mathbf{A}$ in $\mathscr{A}$, the evaluation $e_{\mathbf{A}}$ is an isomorphism. Given that $\mathbf{M}$ is algebraic over $\underline{\mathrm{M}}$ and therefore $e_{\mathrm{A}}$ is already an embedding, this says precisely that, for each $\mathbf{A}$ in $\mathscr{A}$, every morphism from $D(\mathbf{A})$ into $\mathbf{M}$ is an evaluation. We will make frequent use of a simple consequence of this observation.

Let $\mathbf{M}=\langle M ; G, H, R, \mathscr{T}\rangle$ and $\mathbf{M}^{\prime}=\left\langle M ; G^{\prime}, H^{\prime}, R^{\prime}, \mathscr{T}\right\rangle$. Define $\mathscr{X}=\mathbb{U} \mathbb{S}_{c} \mathbb{P} \mathbf{M}$ and $\mathscr{X}^{\prime}=\mathbb{B} \mathbb{S}_{c} \mathbb{P} \mathbf{M}^{\prime}$ and let $D: \mathscr{A} \rightarrow \mathscr{X}, E: \mathscr{X} \rightarrow \mathscr{A}, D^{\prime}: \mathscr{A} \rightarrow \mathscr{X}^{\prime}, E^{\prime}$ : $\mathscr{X}^{\prime} \rightarrow \mathscr{A}$ be the corresponding hom-functors obtained from $\mathbf{M}$ and $\mathbf{M}^{\prime}$ respectively. We say that (the structure on) $\mathbf{M}^{\prime}$ generates a relation $r$ or (partial) operation $h$ if, for all $\mathbf{A} \in \mathscr{A}$, every morphism $\varphi \in \mathscr{X}^{\prime}\left(D^{\prime}(\mathbf{A}), \mathbf{M}^{\prime}\right)$ preserves the relation $r$ or (partial) operation $h$. We say that (the structure on) $\mathbf{M}^{\prime}$ generates (the structure on) $\mathbf{M}$ if $\mathbf{M}^{\prime}$ generates each relation and (partial) operation in $G \cup H \cup R$, that is, if $\mathscr{X}^{\prime}\left(D^{\prime}(\mathbf{A}), \mathbf{M}^{\prime}\right) \subseteq \mathscr{X}(D(\mathbf{A}), \mathbf{M})$. When proving that $\mathbf{M}^{\prime}$ generates $\mathbf{M}$ it is common to prove the following stronger statement: $\mathscr{X}^{\prime}\left(\mathbf{X}^{\prime}, \mathbf{M}^{\prime}\right) \subseteq \mathscr{X}(\mathbf{X}, \mathbf{M})$ whenever $\mathbf{X} \leq \mathbf{M}^{S}$ 
and $\mathbf{X}^{\prime} \leq\left(\mathbf{M}^{\prime}\right)^{s}$ have the same carrier $X \subseteq M^{s}$.

$1.4 \mathbf{M}$-Shift Duality Lemma. If $\mathbf{M}^{\prime}$ generates $\mathbf{M}$ and $\mathbf{M}$ yields a duality on $\mathscr{A}$, then $\mathbf{M}^{\prime}$ also yields a duality on $\mathscr{A}$.

PROOF. Assume that $\mathbf{M}^{\prime}$ generates $\mathbf{M}$ and that $\mathbf{M}$ yields a duality on $\mathscr{A}$. Let $\mathbf{A} \in \mathscr{A} ;$ then $\mathscr{A}(\mathbf{A}, \underline{\mathbf{M}})$ determines substructures $D(\mathbf{A}) \leq \mathbf{M}^{A}$ and $D^{\prime}(\mathbf{A}) \leq\left(\mathbf{M}^{\prime}\right)^{A}$. Thus, from our hypothesis,

$$
E^{\prime} D^{\prime}(\mathbf{A})=\mathscr{X}^{\prime}\left(D^{\prime}(\mathbf{A}), \mathbf{M}^{\prime}\right) \subseteq \mathscr{X}(D(\mathbf{A}), \mathbf{M})=E D(\mathbf{A})=e_{\mathbf{A}}(\mathbf{A}),
$$

and consequently every morphism from $D^{\prime}(\mathbf{A})$ to $\mathbf{M}^{\prime}$ is also an evaluation.

In particular, this lemma tells us that duality is not lost if we add structure to $\mathbf{M}$, nor is it affected if we choose to view some operations as partial operations and some partial operations as relations. As a result, relations alone are sufficient as long as we are interested only in dualities. A list of commonly used constructions for finding relations generated by $\mathbf{M}$ may be found in [16] and [11].

The next step is to find practically verifiable conditions under which a particular choice of $\mathbf{M}$ will yield a duality on $\mathscr{A}$. The structure $\mathbf{M}$ is injective in the category $\mathscr{X}$ if, for every morphism $\alpha: \mathbf{X} \rightarrow \mathbf{M}$ and embedding $\varphi: \mathbf{X} \rightarrow \mathbf{Y}$ in $\mathscr{X}$, there is a morphism $\beta: \mathbf{Y} \rightarrow \mathbf{M}$ such that $\beta \circ \varphi=\alpha$. This notion plays a central role in this study.

1.5 FirST DuAltTy THEOREM. (Davey and Werner $[16,1.8]$ ) The following are equivalent:

(a) M yields a duality on $\mathscr{A}$;

(b) for all $A \in \mathscr{A}$, every morphism $\alpha: D(\mathbf{A}) \rightarrow \mathbf{M}$ extends to an A-ary term function $\tau: M^{A} \rightarrow M$

(c) the following two conditions hold:

(INJ) $M$ is injective with respect to those embeddings in $\mathscr{X}$ which are of the form $D(u): D(\mathbf{B}) \rightarrow D(\mathbf{A})$ where $u: \mathbf{A} \rightarrow \mathbf{B}$ is a surjective homomorphism, that is, for each morphism $\alpha: D(\mathbf{B}) \rightarrow \mathbf{M}$ there exists a morphism $\beta: D(\mathbf{A}) \rightarrow \mathbf{M}$ such that $\beta \circ D(u)=\alpha$,

(CLO) for each $n \in \mathbb{N}$, every morphism $\tau: \mathbf{M}^{n} \rightarrow \mathbf{M}$ is an $n$-ary term function on $\underline{\mathbf{M}}$.

By Lemma 1.1(c), every term function of $\underline{\mathbf{M}}$ is a morphism in $\mathscr{X}$. The condition (CLO) adds the converse: duality requires that the term functions on $\underline{\mathbf{M}}$ must be exactly the morphisms from finite powers of $\mathbf{M}$ into $\mathbf{M}$. Thus (CLO) says precisely 
that the structure on $\mathbf{M}$ determines the clone of term functions on $\underline{\mathbf{M}}$. Given that $M$ is finite, it is easy to see that (CLO) implies the following stronger version (see Davey and Werner [16, 1.8(1)]):

$(\mathrm{CLO})^{\infty}$ for each nonempty set $S$, the morphisms from $\mathbf{M}^{S}$ to $\mathbf{M}$ are precisely the $S$-ary term functions on $\underline{\mathbf{M}}$.

Note that (INJ), which is a special case of the injectivity of $\mathbf{M}$ in $\mathscr{X}$, has been proven in every example to date by actually demonstrating that $\mathbf{M}$ is injective in $\mathscr{X}$. For the finite members of $\mathscr{X}$ we notice that (INJ) and (CLO) follow from the stronger but simpler interpolation condition:

(IC) for each $n \in \mathbb{N}$ and each substructure $\mathbf{X}$ of $\mathbf{M}^{n}$, every morphism $\alpha: \mathbf{X} \rightarrow \mathbf{M}$ extends to a term function $\tau: M^{n} \rightarrow M$ of the algebra M.

Most known dualities can be obtained by verifying (IC) and then invoking the following fundamental theorem of [16].

1.6 SECOND DuAltTy TheOREM. (Davey and Werner $[16,1.16]$ ) Assume that $\mathbf{M}=\langle M ; G, R, \mathscr{T}\rangle$, that is, the structure on $\mathbf{M}$ includes no partial operations, and that $R$ is finite. If (IC) holds, then $\mathbf{M}$ yields a duality on $\mathscr{A}$ and $\mathbf{M}$ is injective in $\mathscr{X}$.

This theorem reflects the spirit of the results that we will seek in this paper. It gives us a simple finitary condition which yields both a dual adjunction between the categories $\mathscr{A}$ and $\mathscr{X}$ and a topological representation of each algebra in $\mathscr{A}$, but it requires us to do no category theory and no topology!

One of the most important and oft used consequences of the Second Duality Theorem is the NU-Duality Theorem. A $(k+1)$-ary term $n\left(x_{1}, \ldots, x_{k+1}\right)$ is called a near-unanimity term on $\underline{\mathbf{M}}$ if $\underline{\mathbf{M}}$ satisfies the identities

$$
n(x, \ldots, x, y) \approx n(x, \ldots, x, y, x) \approx \ldots \approx n(y, x, \ldots, x) \approx x .
$$

A 3-ary near-unanimity term on $\underline{\mathbf{M}}$ is usually called a majority term. For example, on any algebra with an underlying lattice structure, the median

$$
m(x, y, z):=(x \wedge y) \vee(y \wedge z) \vee(z \wedge x)
$$

is a majority term since it satisfies the identities

$$
m(x, x, y) \approx m(x, y, x) \approx m(y, x, x) \approx x .
$$

1.7 NU-DUALITY THEOREM. (Davey and Werner $[16,1.19]$ ) Let $k \geq 2$ and assume that $\underline{\mathbf{M}}$ has a $(k+1)$-ary near-unanimity term. If the structure on $\mathbf{M}$ generates 
all subalgebras of $\underline{\mathbf{M}}^{k}$, then $\mathbf{M}$ yields a duality on $\mathscr{A}$ and $\mathbf{M}$ is injective in $\mathscr{X}$.

Assume that $\langle D, E, e, \varepsilon\rangle$ is a dual adjunction between the categories $\mathscr{A}$ and $\mathscr{X}$. We say that $\langle D, E, e, \varepsilon\rangle$ is a dual equivalence between $\mathscr{A}$ and $\mathscr{X}$ if, for each $\mathbf{A} \in \mathscr{A}$ and $\mathbf{X} \in \mathscr{X}$, both $e_{\mathbf{A}}: \mathbf{A} \rightarrow E D(\mathbf{A})$ and $\varepsilon_{\mathbf{X}}: \mathbf{X} \rightarrow D E(\mathbf{X})$ are isomorphisms. If $\mathscr{A}=\mathbb{\mathbb { S } P} \underline{\mathrm{M}}$, if $\mathscr{X}=\mathbb{\square} \mathbb{S}_{c} \mathbb{P M}$, and if the dual equivalence arises from a duality yielded by $\mathbf{M}$ on $\mathscr{A}$, then we say that $\mathbf{M}$ yields a full duality on $\mathscr{A}$.

As was remarked above, a duality is, in particular, a representation theorem: every algebra $\mathbf{A} \in \mathscr{A}$ is isomorphic to $E(\mathbf{X})=\mathscr{X}(\mathbf{X}, \mathbf{M})$ for some $\mathbf{X} \in \mathscr{X}$. In symbols, $\mathscr{A}=\square E(\mathscr{X})$. If $\mathbf{M}$ yields a full duality on $\mathscr{A}$, then we also have a representation on the topological side: every structure $\mathbf{X} \in \mathscr{X}$ is isomorphic to $D(\mathbf{A})=\mathscr{A}(\mathbf{A}, \underline{\mathbf{M}})$ for some $\mathbf{A} \in \mathscr{A}$. In symbols, $\mathscr{X}=\square D(\mathscr{A})$. As the next lemma shows, in the presence of a duality, such a representation on the topological side is enough to guarantee that the canonical choice, namely $\varepsilon_{\mathbf{X}}: \mathbf{X} \rightarrow D E(\mathbf{X})$, is always an isomorphism, whence the duality between $\mathscr{A}$ and $\mathscr{X}$ is full. At the same time, we shall see that while a duality between $\mathscr{A}$ and $\mathscr{X}$ need not be full, it always yields a dual equivalence between $\mathscr{A}$ and a natural subcategory of $\mathscr{X}$.

1.8 Full Duality Lemma. (i) Assume that $\mathbf{M}$ yields a duality on $\mathscr{A}$. Let $\mathscr{X}^{\prime}=\square D(\mathscr{A})$ and let $E^{\prime}$ and $\varepsilon^{\prime}$ be the restrictions of $E$ and $\varepsilon$ to $\mathscr{X}^{\prime}$. Then $\left\langle D, E^{\prime}, e, \varepsilon^{\prime}\right\rangle$ is a dual equivalence between $\mathscr{A}$ and $\mathscr{X}^{\prime}$.

(ii) The following are equivalent:

(a) $\mathbf{M}$ yields a full duality on $\mathscr{A}$;

(b) $e_{\mathbf{A}}$ is an isomorphism for each $\mathbf{A}$ in $\mathscr{A}$ (that is, $\mathbf{M}$ yields a duality on $\mathscr{A}$ ) and $\mathscr{X}=\square D(\mathscr{A})$;

(c) $\varepsilon_{\mathbf{X}}$ is an isomorphism for each $\mathbf{X}$ in $\mathscr{X}$ and $\mathscr{A}=\square E(\mathscr{X})$.

Moreover, the same is true if $\mathscr{A}$ and $\mathscr{X}$ are replaced by the subcategories $\mathscr{A}_{\mathrm{fin}}$ and $\mathscr{X}_{\text {in }}$ consisting of their respective finite members.

PROOF. Assume that $\mathbf{M}$ yields a duality on $\mathscr{A}$ and let $\mathbf{X} \in \mathscr{X}^{\prime}$. Then there is an $\mathbf{A} \in \mathscr{A}$ and an isomorphism $\varphi: \mathbf{X} \rightarrow D(\mathbf{A})$. Let $u: \mathbf{A} \rightarrow E(\mathbf{X})$ be the corresponding homomorphism given in the definition of a dual adjunction (see Figure 2). By assumption, $e_{\mathbf{A}}$ is an isomorphism, and $E(\varphi)$ is an isomorphism since $\varphi$ is. Consequently $u$ is an isomorphism, whence $D(u)$ is an isomorphism and therefore $\varepsilon_{\mathbf{X}}^{\prime}=D(u)^{-1} \circ \varphi$ is an isomorphism. Thus (i) holds.

Part (ii) follows almost at once from (i). It is trivial that (a) implies both (b) and (c); that (b) implies (a) is a consequence of (i); and (c) implies (a) by symmetry. Note that these arguments apply equally well to the finitary version of the lemma. 


\section{Full duality and the dual category}

In this section we will investigate when the duality given by a structure $\mathbf{M}$ can be upgraded to a full duality on $\mathscr{A}$. A brief review of the last section reveals a rich source of raw materials out of which we might begin to build full dualities. If $\mathbf{M}$ yields a duality on $\mathscr{A}$, then Part (i) of the Full Duality Lemma (1.8) tells us that $D$ and $E$ yield a dual equivalence between $\mathscr{A}$ and the subcategory $\square D(\mathscr{A})$ of $\mathscr{X}$. By itself this fact is of rather limited value since in practice we have no way to identify members of $\square D(\mathscr{A})$ within $\mathscr{X}$. But now the $\mathbf{M}$-Shift Duality Lemma (1.4) comes into play. If $\mathbf{M}$ yields a duality on $\mathscr{A}$, then it will do so regardless of whether its operations and partial operations are viewed as members of $G \cup H$ or they are viewed, via their graphs, as relations in $R$. Also, duality will not be affected by the addition to $G, H$ and $R$ of new (partial) operations and relations which are algebraic over $\underline{\mathbf{M}}$. This means that we have considerable freedom to modify a choice of $\mathbf{M}$, which yields a duality on $\mathscr{A}$, without jeopardizing that duality. By adding new (partial) operations to $G \cup H$ and transferring relations which are graphs of operations from $R$ to $G \cup H$ we will be able to eliminate structures from $\mathscr{X}$ which are not in $\nabla D(\mathscr{A})$. Ultimately we will seek to add enough structure to $\mathbf{M}$ to obtain $\mathscr{X}=\square D(\mathscr{A})$.

We begin by examining the structure imposed on the category $\mathscr{X}$ by the dual adjunction arising from an arbitrary choice of the generating structure $\mathbf{M}$. Our analysis depends on two different descriptions of the dual category $D D(\mathscr{A})$ which will give us a basic conceptual model to tell when $\mathbf{M}$ yields a full duality on $\mathscr{A}$. This section constitutes a revised exposition of Lemma 2.14 to Theorem 2.26 of Clark and Krauss [5].

Let $I$ be an arbitrary nonempty set, $\mathbf{B}$ a subalgebra of $\underline{\mathbf{M}}^{I}$ and $h: \mathbf{B} \rightarrow \underline{\mathbf{M}}$ a homomorphism, that is, $h$ is an algebraic I-ary partial operation on $\underline{\mathbf{M}}$. Just as we do in the finitary case, we may extend $h$ pointwise to an $I$-ary partial operation $\mathbf{h}$ on any power $M^{S}$ of $M$. For each $s \in S$, let $\pi_{S}: M^{S} \rightarrow M$ denote the sth projection given by $\pi_{s}(y)=y(s)$ for each $y \in M^{S}$. Then the domain of the extension $\mathbf{h}$ is

$$
\operatorname{dom}(\mathbf{h})=\left\{\mathbf{x} \in\left(M^{S}\right)^{I} \mid \pi_{s} \circ \mathbf{x} \in B \text { for all } s \in S\right\} \subseteq\left(M^{S}\right)^{I}
$$

and $\mathbf{h}: \operatorname{dom}(\mathbf{h}) \rightarrow \underline{\mathbf{M}}^{s}$ is defined by $(\mathbf{h}(\mathbf{x}))(s)=h\left(\pi_{s} \circ \mathbf{x}\right)$ for $\mathbf{x} \in \operatorname{dom}(\mathbf{h})$. As is customary, we say that a subset $X$ of $M^{S}$ is closed under $h$ provided $\mathbf{h}(\mathbf{x}) \in X$ whenever $\mathbf{x} \in \operatorname{dom}(\mathbf{h})$ and $\mathbf{x}(i) \in X$ for each $i \in I$. We shall say that $X$ is hom-closed (in $M^{S}$ ) if, for each nonempty set $I$, the set $X$ is closed under every algebraic $I$-ary partial operation $h$ on $\underline{\mathbf{M}}$.

For an arbitrary nonempty set $S$, the subalgebra of $\underline{\mathbf{M}}^{M^{s}}$ ( $\mathscr{A}$-freely) generated by the projections $\left\{\pi_{s} \mid s \in S\right\}$ is called the algebra of S-ary term functions on $\underline{\mathbf{M}}$ and is denoted by $\mathbf{T}_{s}$. We say that a subset $X$ of $M^{S}$ is term-closed (in $M^{S}$ ) if for all $y \in M^{S} \backslash X$ there exist $S$-ary term functions $\sigma, \tau: M^{S} \rightarrow M$ on $\underline{\mathbf{M}}$ that agree on 
$X$ but not at $y$. Thus $X$ is term-closed in $M^{s}$ provided it is an intersection of the equalizer sets

$$
\mathrm{Eq}(\sigma, \tau)=\left\{x \in M^{S} \mid \sigma(x)=\tau(x)\right\}
$$

of pairs $(\sigma, \tau)$ of $S$-ary term functions from $\mathbf{T}_{S}$.

Despite their disparate origins, these two notions of closure prove to be equivalent. The proof of this fact requires a modification of the embedding $\varepsilon_{\mathbf{X}}$. Let $S \neq \emptyset$, let $\mathbf{X} \leq \mathbf{M}^{S}$ and let $\mathbf{T}_{S} \uparrow_{\mathbf{x}} \leq \underline{\mathbf{M}}^{X}$ be the restriction of the algebra of $S$-ary term functions to $X$ :

$$
T_{S} \uparrow_{X}=\left\{\tau \uparrow_{X}: X \rightarrow M \mid \tau \in T_{S}\right\} .
$$

Define the evaluation map $\delta_{\mathbf{X}}: X \rightarrow D\left(\mathbf{T}_{S} \uparrow_{X}\right)$ by $\delta_{\mathbf{X}}(x)\left(\tau \uparrow_{X}\right)=\tau(x)$ for $x \in X$ and $\tau \in T_{s}$. (See Figure 4.)

$$
\mathbf{T}_{S} \subseteq \underline{\mathbf{M}}^{M^{S}}
$$

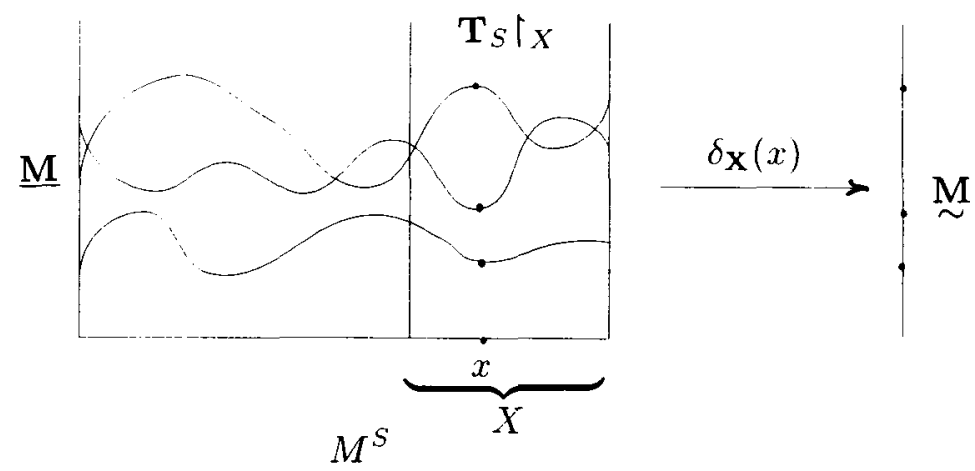

FIGURE 4

In the proof of (b) implies (c) below, notice the schizophrenic nature of $X$, which acts simultaneously as a substructure of a power of $\mathbf{M}$ and as the exponent of a power of $\underline{\mathbf{M}}$ !

2.1 Closure TheOREM. If $S \neq \emptyset$ and $\emptyset \neq X \subseteq M^{S}$, then the following are equivalent:

(a) $X$ is term-closed in $M^{s}$;

(b) $X$ is hom-closed in $M^{s}$;

(c) every homomorphism $u: \mathbf{T}_{S} \uparrow_{X} \rightarrow \underline{\mathbf{M}}$ is an evaluation map;

(d) $\mathbf{X} \in \mathscr{X}$ (that is, $\mathbf{X}$ is a closed substructure of $\mathbf{M}^{s}$ ) and the map $\delta_{\mathbf{X}}: \mathbf{X} \rightarrow$ $D\left(\left.\mathbf{T}_{S}\right|_{X}\right)$ is an isomorphism.

PROOF. Assume that $X$ is term-closed in $M^{S}$. Let $\mathbf{B}$ be a subalgebra of $\underline{\mathbf{M}}^{I}$, let $h: \mathbf{B} \rightarrow \underline{\mathbf{M}}$ and let $\mathbf{x} \in X^{I}$ be in the domain of $\mathbf{h}$. We will show that $\mathbf{h}(\mathbf{x})$ is in the term 
closure of $X$. Since $\mathbf{T}_{S}$ is generated by $\left\{\pi_{s} \mid s \in S\right\}$, each member of $T_{S}$ is of the form $\tau^{\left(\mathbf{T}_{s}\right)}\left(\pi_{s_{1}}, \ldots, \pi_{s_{n}}\right)$ where $\tau$ is an $n$-ary term. Assume, then, that $\tau^{\left(\mathbf{T}_{s}\right)}\left(\pi_{s_{1}}, \ldots, \pi_{s_{n}}\right)$ and $\sigma^{\left(T_{s}\right)}\left(\pi_{s_{1}}, \ldots, \pi_{s_{n}}\right)$ agree on $X$. Observe that

$$
\tau^{\mathbf{B}}\left(\pi_{s_{1}} \circ \mathbf{x}, \ldots, \pi_{s_{n}} \circ \mathbf{x}\right), \sigma^{\mathbf{B}}\left(\pi_{s_{1}} \circ \mathbf{x}, \ldots, \pi_{s_{n}} \circ \mathbf{x}\right) \in M^{\prime}
$$

and that, for any $i \in I$,

$$
\begin{aligned}
& \tau^{\mathbf{B}}\left(\pi_{s_{1}} \circ \mathbf{x}, \ldots, \pi_{s_{n}} \circ \mathbf{x}\right)(i)=\tau^{\underline{\mathbf{M}}}\left(\pi_{s_{1}} \mathbf{x}(i), \ldots, \pi_{s_{n}} \mathbf{x}(i)\right) \\
& =\tau^{\left(\mathbf{T}_{s}\right)}\left(\pi_{s_{1}}, \ldots, \pi_{s_{n}}\right)(\mathbf{x}(i))=\sigma^{\left(\mathbf{T}_{s}\right)}\left(\pi_{s_{1}}, \ldots, \pi_{s_{n}}\right)(\mathbf{x}(i)) \\
& =\sigma^{\underline{\mathbf{M}}}\left(\pi_{s_{1}} \mathbf{x}(i), \ldots, \pi_{s_{n}} \mathbf{x}(i)\right)=\sigma^{\mathbf{B}}\left(\pi_{s_{1}} \circ \mathbf{x}, \ldots, \pi_{s_{n}} \circ \mathbf{x}\right)(i)
\end{aligned}
$$

from which we conclude that $\tau^{\mathbf{B}}\left(\pi_{s_{1}} \circ \mathbf{x}, \ldots, \pi_{s_{n}} \circ \mathbf{x}\right)=\sigma^{\mathbf{B}}\left(\pi_{s_{1}} \circ \mathbf{x}, \ldots, \pi_{s_{n}} \circ \mathbf{x}\right)$. Thus

$$
\begin{aligned}
& \tau^{\left(\mathbf{T}_{s}\right)}\left(\pi_{s_{1}}, \ldots, \pi_{s_{n}}\right)(\mathbf{h}(\mathbf{x}))=\tau^{\underline{\mathbf{M}}}\left(\mathbf{h}(\mathbf{x})\left(s_{1}\right), \ldots, \mathbf{h}(\mathbf{x})\left(s_{n}\right)\right) \\
& =\tau^{\underline{\mathbf{M}}}\left(h\left(\pi_{s_{1}} \circ \mathbf{x}\right), \ldots, h\left(\pi_{s_{n}} \circ \mathbf{x}\right)\right)=h \tau^{\mathbf{B}}\left(\left(\pi_{s_{1}} \circ \mathbf{x}\right), \ldots,\left(\pi_{s_{n}} \circ \mathbf{x}\right)\right) \\
& =h \sigma^{\mathbf{B}}\left(\left(\pi_{s_{1}} \circ \mathbf{x}\right), \ldots,\left(\pi_{s_{n}} \circ \mathbf{x}\right)\right)=\sigma^{\underline{\mathbf{M}}}\left(h\left(\pi_{s_{1}} \circ \mathbf{x}\right), \ldots, h\left(\pi_{s_{n}} \circ \mathbf{x}\right)\right) \\
& =\sigma^{\underline{\mathbf{M}}}\left(\mathbf{h}(\mathbf{x})\left(s_{1}\right), \ldots, \mathbf{h}(\mathbf{x})\left(s_{n}\right)\right)=\sigma^{\left(\mathbf{T}_{s}\right)}\left(\pi_{s_{1}}, \ldots, \pi_{s_{n}}\right)(\mathbf{h}(\mathbf{x})) .
\end{aligned}
$$

Since $\mathbf{h}(\mathbf{x})$ is in the term-closure of $X$, it is in $X$. Thus (a) implies (b).

Assume that (b) holds. In order to prove (c), we apply (b) to the closure of $X$ under $u$. Let $\mathbf{B}=\mathbf{T}_{S} \uparrow \leq \leq \underline{\mathbf{M}}^{X}$ and let $\mathbf{i} \in\left(M^{S}\right)^{X}$ be the inclusion map $\mathbf{i}(x)=x$. Then $\mathbf{i} \in \operatorname{dom}(\mathbf{u})$ since $\pi_{s} \circ \mathbf{i}=\pi_{s} \uparrow_{X}$, for each $s \in S$. By hom-closure, $\mathbf{u}(\mathbf{i}) \in X$. We check that $u$ is exactly the evaluation $\delta_{\mathbf{X}}(\mathbf{u}(\mathbf{i}))$. Since both are homomorphisms, it is sufficient to see that they agree on each generator $\pi_{s} \uparrow_{X}$ where $s \in S$ :

$$
\delta_{\mathbf{X}}(\mathbf{u}(\mathbf{i}))\left(\pi_{s} \uparrow_{X}\right)=\pi_{s}(\mathbf{u}(\mathbf{i}))=\mathbf{u}(\mathbf{i})(s)=u\left(\pi_{s} \circ \mathbf{i}\right)=u\left(\pi_{s} \uparrow_{X}\right) .
$$

We now show that (c) implies (a). Suppose $z \in M^{S}$ and any two $S$-ary term functions which agree on $X$ also agree at $z$. Then the map $u: T_{S} \uparrow_{X} \rightarrow M$ defined by $u\left(\tau \uparrow_{X}\right)=\tau(z)$ is a well-defined homomorphism. By (c) there is an $x \in X$ such that $u$ is evaluation at $x$. Thus for all $s \in S$ we have

$$
z(s)=\pi_{s}(z)=u\left(\pi_{s} \uparrow X\right)=\pi_{s}(x)=x(s)
$$

and consequently $z=x \in X$. Hence $X$ is term closed.

Since it is trivial that (d) implies (c), to complete the proof it suffices to prove that (a) and (b) together imply (d). Topological closure is an immediate consequence of termclosure, and closure under algebraic (partial) operations is an immediate consequence of hom-closure. Thus $\mathbf{X} \in \mathscr{X}$. The proof that $\delta_{\mathbf{X}}$ is an embedding is routine, and is exactly the same as the proof of Lemma 1.5 of Davey and Werner [16]. 
2.2 LEMMA. (Clark and Krauss [5, 2.17]) D(A) is term-closed (= hom-closed) in $M^{A}$ for all $\mathbf{A} \in \mathscr{A}$.

PROOF. Let $y \in M^{A}$ and assume that any two term functions in $\mathbf{T}_{A}$ that agree on $D(\mathbf{A})$ also agree at $y$. Let $f$ be an $n$-ary operation in the type of $\mathscr{A}$ and let $a_{1}, \ldots, a_{n} \in \mathscr{A}$. For any $x \in D(\mathbf{A})$, the maps $\sigma=\pi_{f\left(a_{1}, \ldots, a_{n}\right)}$ and $\tau=f\left(\pi_{a_{1}}, \ldots, \pi_{a_{n}}\right)$ agree at $x$ :

$$
\begin{aligned}
\sigma(x) & =\pi_{f\left(a_{1}, \ldots, a_{n}\right)}(x)=x\left(f\left(a_{1}\right), \ldots, x\left(a_{n}\right)\right)=f\left(x\left(a_{1}\right), \ldots, x\left(a_{n}\right)\right) \\
& =f\left(\pi_{a_{1}}(x), \ldots, \pi_{a_{n}}(x)\right)=f\left(\pi_{a_{1}}, \ldots, \pi_{a_{n}}\right)(x)=\tau(x) .
\end{aligned}
$$

Consequently, $\sigma$ and $\tau$ also agree at $y$. Thus

$$
\begin{aligned}
& y\left(f\left(a_{1}, \ldots, a_{n}\right)\right)=\pi_{f\left(a_{1}, \ldots, a_{n}\right)}(y)=\sigma(y)=\tau(y)= \\
& \quad f\left(\pi_{a_{1}}, \ldots, \pi_{a_{n}}\right)(y)=f\left(\pi_{a_{1}}(y), \ldots, \pi_{a_{n}}(y)\right)=f\left(y\left(a_{1}\right), \ldots, y\left(a_{n}\right)\right),
\end{aligned}
$$

whence $y \in D(\mathbf{A})$.

If $\mathbf{M}$ yields a duality on $\mathscr{A}$, then Part (i) of the Full Duality Lemma (1.8) tells us that $D$ and $E$ (restricted to $\square D(\mathscr{A})$ ) yield a dual equivalence between the categories $\mathscr{A}$ and $\square D(\mathscr{A})$. As an immediate consequence of these results we get two fundamental descriptions of the dual category $D D(\mathscr{A})$ that will be used throughout the rest of this paper.

2.3 Corollary. (Clark and Krauss [5, 2.26]) Let $\mathbf{X} \in \mathscr{X}$. Then $\mathbf{X} \in \mathbb{D} D(\mathscr{A})$ if and only if $\mathbf{X}$ is isomorphic to a term-closed (= hom-closed) subset of $\mathbf{M}^{s}$ for some nonempty set $S$.

The content of the last three results is summarized in Figure 5.

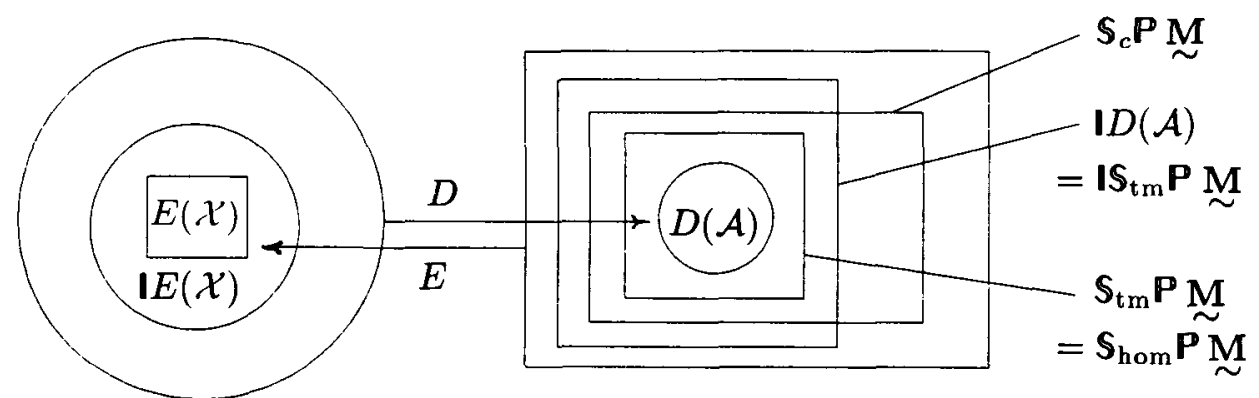

$$
\mathcal{A}=\text { ISP } \underline{\mathrm{M}} \quad \mathcal{X}=\text { IS }_{\mathrm{c}} \mathbf{P} \underset{\sim}{\mathrm{M}}
$$

FIGURE 5. subcategories of $\mathscr{X}$ under a dual adjunction. Here $\mathbb{S}_{\mathrm{tm}}$ and $\mathbb{S}_{\text {hom }}$ denote, respectively, term-closed and hom-closed subsets. 
The following variant of the First Duality Theorem (1.5) is more exactly suited to our needs. Here we have replaced (INJ) with the injectivity condition (INJ)' which is expressed fully in terms of the category $\mathscr{X}$.

2.4 THIRD DUALITY THEOREM. (Clark and Krauss [5, 2.25]) The following are equivalent:

(a) M yields a duality on $\mathscr{A}$;

(b) if $\mathbf{X}$ is a term-closed substructure of $\mathbf{M}^{s}$ for some $S \neq \emptyset$, then $E(\mathbf{X})=\mathbf{T}_{S} \uparrow_{X}$, that is, every morphism $\alpha: \mathbf{X} \rightarrow \mathbf{M}$ extends to an $S$-ary term function $\tau: \mathbf{M}^{S} \rightarrow \mathbf{M}$;

(c) the following conditions hold:

$(\mathrm{INJ})^{\prime} \mathbf{M}$ is injective with respect to term-closed sets $\mathbf{X} \subseteq \mathbf{M}^{S}$ (with $\left.S \neq \emptyset\right)$ and their inclusion maps, that is, each morphism $\alpha: \mathbf{X} \rightarrow \mathbf{M}$ extends to a morphism $\beta: \mathbf{M}^{S} \rightarrow \mathbf{M}$,

(CLO) for each $n \in \mathbb{N}$, every morphism $\tau: \mathbf{M}^{n} \rightarrow \mathbf{M}$ is an $n$-ary term function on $\underline{\mathbf{M}}$.

Moreover, the same is true if $\mathscr{A}$ is replaced by $\mathscr{A}_{\mathrm{fin}}$ in (a) and $S$ is restricted to finite sets in (b) and (c).

Proof. Assume that $\mathbf{M}$ yields a duality on $\mathscr{A}$ and that $\mathbf{X}$ is term-closed in $\mathbf{M}^{S}$. The dual adjunction yields the triangles in Figure 6, where $i: \mathbf{T}_{s} \uparrow_{X} \rightarrow E(\mathbf{X})$ is the inclusion map. If $\mathbf{M}$ yields a duality on $\mathscr{A}$, then $e_{\left(\left.\mathbf{T}_{s}\right|_{X)}\right)}$ is surjective. By the Closure Theorem $(2.1), \delta_{\mathbf{X}}$ is an isomorphism and hence $E\left(\delta_{\mathbf{X}}\right)$ is an isomorphism. By the left-hand triangle, the inclusion map $i$ is surjective and we have $E(\mathbf{X})=\mathbf{T}_{S} \uparrow x$. Hence, (a) implies (b).
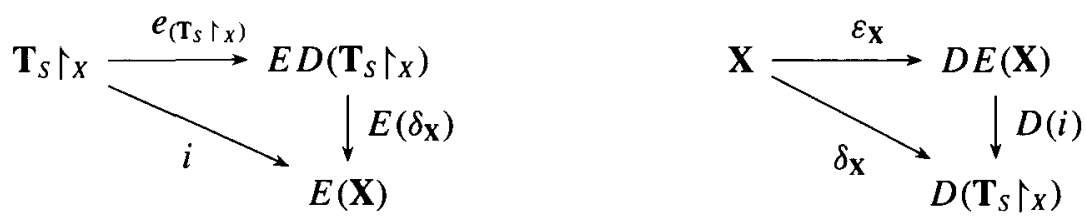

FIGURE 6. An application of Figure 2

Since (b) implies (c) trivially, it remains to prove that (c) implies (a). We verify the second condition of the First Duality Theorem (1.5). Let $\mathbf{A} \in \mathscr{A}$ and let $\alpha: D(\mathbf{A}) \rightarrow$ M. By Lemma 2.2, the set $D(\mathbf{A})$ is term-closed and therefore, by (INJ)' it follows that $\alpha$ extends to a morphism $\beta: \mathbf{M}^{A} \rightarrow \mathbf{M}$ which, since (CLO) implies (CLO) ${ }^{\infty}$, is an $A$-ary term function on $\underline{\mathbf{M}}$.

To prove the finitary version ('Moreover, ... '), first observe that the same argument again establishes that (a) implies (b), and that now (b) and (c) are identical. To prove 
that (b) implies (a), consider $n \in \mathbb{N}$ and $\eta: D(\mathbf{A}) \rightarrow \mathbf{M}$ where $\mathbf{A} \leq \underline{M}^{n}$. By Lemma (2.2), the set $D(\mathbf{A})$ is term-closed and consequently $\beta$ extends to an $A$-ary term function $\tau$. Thus there are $a, b, c, \ldots \in A$ such that for all $x \in D(\mathbf{A})$, we have that

$$
\begin{aligned}
\eta(x) & =\tau\left(\pi_{a}, \pi_{b}, \pi_{c}, \ldots\right)(x)=\tau(x(a), x(b), x(c), \ldots) \\
& =x(\tau(a, b, c, \ldots))=e_{\mathbf{A}}(\tau(a, b, c, \ldots))(x) .
\end{aligned}
$$

Thus $e_{\mathrm{A}}$ is surjective and we have (a).

In practice we will normally begin with a duality which we seek to upgrade to a full duality. Our characterization of the dual category tells us just what will be required to do this.

2.5 FULL DUALITY THEOREM. If $\mathbf{M}$ yields a duality on $\mathscr{A}$, then the following are equivalent:

(a) M yields a full duality on $\mathscr{A}$;

(b) $\mathscr{X}=\mathbb{\square}(\mathcal{A})$;

(c) every closed substructure of a power of $\mathbf{M}$ is isomorphic to a term-closed (= hom-closed) substructure of a power of $\mathbf{M}$.

Moreover, the same is true if $\mathscr{A}$ and $\mathscr{X}$ are replaced by $\mathscr{A}_{\mathrm{fin}}$ and $\mathscr{X}_{\text {fin }}$ in (a) and (b), and (c) is restricted to finite powers of $\mathbf{M}$.

PRoOF. Combine Part (ii) of the Full Duality Lemma (1.8) with Corollary 2.3.

This theorem tells us that the duality, which $\mathbf{M}$ yields on $\mathscr{A}$, will be full exactly when we have the cleaner arrangement of subcategories of $\mathscr{X}$ illustrated in Figure 7. We turn next to the question of how and when this will be the case.

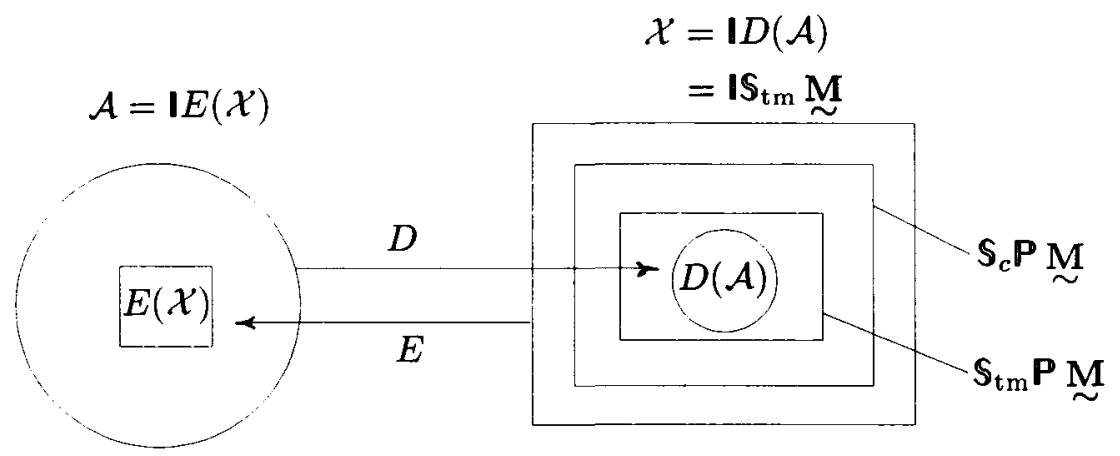

FIGURE 7. Subcategories of $\mathscr{X}$ under a full duality. 


\section{Strong duality and the role of injectivity}

Assume that $\mathbf{M}$ yields a duality on $\mathscr{A}$. To establish that $\mathbf{M}$ yields a full duality on $\mathscr{A}$, the Full Duality Theorem (2.5) says that we must find, for each nonempty set $S$ and each closed substructure $\mathbf{X}$ of $\mathbf{M}^{S}$, a nonempty set $T$ and term-closed (= homclosed) substructure $\mathbf{Y}$ of $\mathbf{M}^{T}$ such that $\mathbf{X}$ is isomorphic to $\mathbf{Y}$. Now this seemingly daunting assignment is actually carried out in every known example of full duality by establishing that the natural candidate $\mathbf{Y}=\mathbf{X}$ will suffice, that is, that every closed substructure of a power of $\mathbf{M}$ is either term-closed or hom-closed. When this is true we say that $\mathbf{M}$ yields a strong duality on $\mathscr{A}$. While proving strong duality is the most natural way to establish a full duality, it also provides a valuable enhancement of full duality which will prove useful in many ways. In this section we will see that strong duality implies the injectivity of $\mathbf{M}$ in $\mathscr{X}$, and, conversely, that a full duality for which $\mathbf{M}$ is injective in $\mathscr{X}$ is necessarily strong. The injectivity of $\mathbf{M}$ considerably strengthens the dual equivalence between $\mathscr{A}$ and $\mathscr{X}$. We will prove several theorems giving different criteria for establishing a strong duality. In particular we will see how the criteria become simpler and more direct as the structure on $\mathbf{M}$ becomes more tractable. Note that in Figure 7 the duality is strong when the two inner boxes on the right are really one.

Once we have a structure $\mathbf{M}$ which yields a strong duality on $\mathscr{A}$, we often wish to modify the structure on $\mathbf{M}$ without destroying the strong duality.

3.1 M-Shift Strong Duality Lemma. Assume that $\mathbf{M}=\langle M ; G, H, R, \mathscr{T}\rangle$ yields a strong duality on $\mathscr{A}$. Then $\mathbf{M}^{\prime}$ will also yield a strong duality on $\mathscr{A}$ if it is obtained from $\mathbf{M}$ by:

(a) enlarging $G, H$ or $R$, or

(b) deleting members of $G$ or $H$ which can be obtained as compositions of the remaining members of $G$ and $H$ and the projection mappings.

Moreover, if $\mathbf{M}^{\prime}$ yields a duality on $\mathscr{A}$ and is obtained from $\mathbf{M}$ by

(c) deleting members of $R$, or

(d) deleting members of $H$ which have an extension in $G$ or $H$, then $\mathbf{M}^{\prime}$ will also yield a strong duality on $\mathscr{A}$.

Proof. Use the M-Shift Duality Lemma (1.4) to show that duality is not disturbed in (a) and (b), and the hom-closure definition of strong duality to show that strong duality is not disturbed in all four cases.

Assume that $\mathbf{M}$ yields a duality on $\mathscr{A}_{\text {fin }}$, that is, $e_{\mathbf{A}}: \mathbf{A} \rightarrow E D(\mathbf{A})$ is an isomorphism for every finite algebra $\mathbf{A}$ in $\mathscr{A}$. We say that $\mathbf{M}$ yields a full duality on 
$\mathscr{A}_{\text {fin }}$ if $\varepsilon_{\mathbf{X}}: \mathbf{X} \rightarrow D E(\mathbf{X})$ is an isomorphism for every finite $\mathbf{X}$ in $\mathscr{X}$ and that $\mathbf{M}$ yields a strong duality on $\mathscr{A}_{\text {fin }}$ if every (closed) substructure of a finite power of $\mathbf{M}$ is term-closed (= hom-closed).

It was pointed out by Clark and Krauss [5] that every example of a full duality known to them was strong. Davey and Werner [16] noted that in every full duality known to them the structure $M$ was injective in $\mathscr{X}$. As it happens, these two enhancements of full duality turn out to be equivalent. (The theorem below strengthens the result announced in Davey $[11,3.4]$.)

3.2 FIRST STRONG DUALITY THEOREM. M yields a strong duality on $\mathscr{A}$ if and only if $\mathbf{M}$ yields a full duality on $\mathscr{A}$ and is injective in $\mathscr{X}$. The corresponding result holds at the finite level, that is, $\mathbf{M}$ yields a strong duality on $\mathscr{A}_{\text {fin }}$ if and only if $\mathbf{M}$ yields a full duality on $\mathscr{A}_{\text {fin }}$ and is injective in $\mathscr{X}_{\text {fin }}$.

PROOF. Assume $\mathbf{M}$ yields a strong duality on $\mathscr{A}$. Then $\mathbf{M}$ yields a full duality on $\mathscr{A}$ by the Full Duality Theorem (2.5). To prove injectivity, let $\varphi: \mathbf{Y} \rightarrow \mathbf{M}^{S}$ be an embedding and let $\alpha: \mathbf{Y} \rightarrow \mathbf{M}$ be a morphism in $\mathscr{X}$. Then the image of $\mathbf{Y}$ under $\varphi$ is a closed substructure $\mathbf{X}$ of $\mathbf{M}^{S}$ and we have $\alpha \varphi^{-1}: \mathbf{X} \rightarrow \mathbf{M}$. By strong duality $\mathbf{X}$ is term-closed in $\mathbf{M}^{S}$, and by the Third Duality Theorem (2.4), the map $\alpha \varphi^{-1}$ extends to an $S$-ary term function $\tau$ on $\underline{\mathbf{M}}$. Thus $\tau \circ \varphi: Y \rightarrow \mathbf{M}$ is the required extension of $\alpha$.

Conversely, suppose $\mathbf{M}$ yields a full duality on $\mathscr{A}$ and is injective in $\mathscr{X}$. Let $S \neq \emptyset$, let $\mathbf{X} \leq \mathbf{M}^{S}$ and let $y \in M^{S} \backslash X$. Denote by $\mathbf{Y}$ the closed substructure of $\mathbf{M}^{S}$ generated by $X \cup\{y\}$, that is, $\mathbf{Y}$ is the intersection of all closed substructures of $\mathbf{M}^{s}$ which contain $X \cup\{y\}$. Let $\iota: \mathbf{X} \rightarrow \mathbf{Y}$ be the inclusion map. By injectivity, each member of $E(\mathbf{X})$ has an extension in $E(\mathbf{Y})$, that is to say, $E(\iota): E(\mathbf{Y}) \rightarrow E(\mathbf{X})$ is surjective. Since $\mathbf{X} \neq \mathbf{Y}$, the inclusion $\iota$ is not an isomorphism and hence $E(\iota)$ is not an isomorphism. Thus $E(l)$ is not an embedding and we must have $\sigma, \tau \in E(\mathbf{Y})$ with $\sigma \neq \tau$ but $\sigma \uparrow_{X}=\tau \uparrow_{X}$. Suppose that $\sigma(y)=\tau(y)$. Then $X \cup\{y\} \subseteq \mathrm{Eq}(\sigma, \tau)$, whence $Y \subseteq \mathrm{Eq}(\sigma, \tau)$, by the definition of $\mathbf{Y}$, since $\mathrm{Eq}(\sigma, \tau)$ is a closed substructure of $\mathbf{M}^{S}$. Thus $Y=\mathrm{Eq}(\sigma, \tau)$, which contradicts the fact that $\sigma \neq \tau$. Hence, $\sigma(y) \neq \tau(y)$. Finally, $\sigma$ and $\tau$ extend to morphisms on $\mathbf{M}^{s}$ by injectivity, and these are $S$-ary term functions by Part (b) of the Third Duality Theorem (2.4).

The same proof, restricted to finite powers of $\mathbf{M}$, yields the finite version.

In the presence of full duality, the injectivity of $\mathbf{M}$ in $\mathscr{X}$ has immediate algebraic consequences. It says precisely that embeddings go to surjections under $E$, but in this setting it says more. (See the Dual Adjunction Theorem (1.3).)

3.3 LEMMA. Assume that $\mathbf{M}$ yields a full duality on $\mathscr{A}$. Then the following are equivalent: 
(a) $\mathbf{M}$ is injective in $\mathscr{X}$;

(b) $\varphi: \mathbf{X} \rightarrow \mathbf{Y}$ is an embedding in $\mathscr{X}$ (if and) only if $E(\varphi): E(\mathbf{Y}) \rightarrow E(\mathbf{X})$ is a surjection in $\mathscr{A}$;

(c) $u: \mathbf{A} \rightarrow \mathbf{B}$ is a surjection in $\mathscr{A}$ if (and only if) $D(u): D(\mathbf{B}) \rightarrow D(\mathbf{A})$ is an embedding in $\mathscr{X}$.

PROOF. The equivalence of (a) and (b) is an immediate consequence of the definitions.

To prove that (b) implies (c), we use the fact that each $e_{\mathrm{A}}$ is an isomorphism. Let $D(u)$ be an embedding. By (b), the map $E D(u)$ is surjective. From Figure 1 (since $D$ and $E$ are dually adjoint) we have that $u=e_{\mathrm{B}}^{-1} \circ E D(u) \circ e_{\mathrm{A}}$ is also surjective.

To prove that (c) implies (b), we use the fact that each $\varepsilon_{\mathbf{X}}$ is an isomorphism. Let $\varphi$ be an embedding. From Figure 1 we have that $D E(\varphi)=\varepsilon_{\mathbf{Y}} \circ \varphi \circ \varepsilon_{\mathbf{X}}^{-1}$ is also an embedding. By (c), we conclude that $E(\varphi)$ is surjective.

The First Duality Theorem (1.5) and the Third Duality Theorem (2.4) say that many instances of the injectivity of $\mathbf{M}$ in $\mathscr{X}$ must hold once we have a duality. If duality is to be established by means of the Second Duality Theorem (1.6), then $\mathbf{M}$ must, indeed, be injective in $\mathscr{X}$. Nevertheless, it is not known whether or not the injectivity of $\mathbf{M}$ in $\mathscr{X}$ is actually required for $\mathbf{M}$ to yield a full duality. In the view of the authors, this is one of the most fundamental open question in the foundations of duality theory.

3.4 PROBLEM. Does there exist a finite algebra $\underline{\mathbf{M}}$ and a choice of $\mathbf{M}$ such that $\mathbf{M}$ yields a duality on $\mathscr{A}=\square \mathbb{} \mathbb{P} \underline{\mathbf{M}}$ which is full but not strong, or equivalently, such that $\mathbf{M}$ yields a full duality on $\mathscr{A}$ with $\mathbf{M}$ non-injective in $\mathscr{X}$ ?

In every known full duality, $\mathbf{M}$ is injective in $\mathscr{X}$. The corresponding statement about $\underline{M}$ is false: let $\underline{\mathbf{M}}$ be the 4-element chain as a Heyting algebra - see Theorem (5.2). In many instances we come to duality with prior information about the injectivity of $\underline{\mathbf{M}}$ in $\mathscr{A}$. The injectivity of $\underline{\mathbf{M}}$ in $\mathscr{A}$ says precisely that $D$ maps embeddings to surjections, and from this we obtain the following analog of Lemma 3.3 for $\underline{\mathbf{M}}$ by the exact dual argument.

3.5 LEMMA. Assume that $\mathbf{M}$ yields a full duality on $\mathscr{A}$. Then the following are equivalent:

(a) $\mathbf{M}$ is injective in $\mathscr{A}$;

(b) $u: \mathbf{A} \rightarrow \mathbf{B}$ is an embedding in $\mathscr{A}$ (if and) only if $D(u): D(\mathbf{B}) \rightarrow D(\mathbf{A})$ is a surjection in $\mathscr{X}$;

(c) $\varphi: \mathbf{X} \rightarrow \mathbf{Y}$ is a surjection in $\mathscr{X}$ if (and only if) $E(\varphi): E(\mathbf{Y}) \rightarrow E(\mathbf{X})$ is an embedding in $\mathscr{A}$. 
PROOF. The proof is the same as that of Lemma 3.3. The equivalence of (a) and (b) is an immediate consequence of the definitions. To prove that (b) implies (c), use the fact that each $\varepsilon_{\mathbf{X}}$ is an isomorphism. To prove that (c) implies (a), use the fact that each $e_{\mathrm{A}}$ is an isomorphism.

If $\mathbf{M}=\langle M ; G, R, \mathscr{T}\rangle$, that is, every operation of $\mathbf{M}$ is total rather than partial, then we call $\mathbf{M}$ a total structure. In this case we find that we can establish strong duality by checking term-closure for just the substructures of finite powers of $\mathbf{M}$. We state this condition explicitly in the next theorem, where the equivalence of (a) and (c) is a reformulation of Lemma 2.33 of Clark and Krauss [5]. On a first reading, it is easy to miss the point where this argument depends on the absence of partial operations in $\mathbf{M}$.

3.6 SECOND STRONG DUALITY THEOREM. If $\mathbf{M}$ is a total structure which yields a duality on $\mathscr{A}$, then the following are equivalent:

(a) M yields a strong duality on $\mathscr{A}$;

(b) M yields a strong duality on $\mathscr{A}_{\text {fin }}$;

(c) $\mathbf{M}$ satisfies the Finite Term Closure condition-

(FTC) If $\mathbf{X}$ is a substructure of $\mathbf{M}^{n}$ for some $n \in \mathbb{N}$ and $y \in M^{n} \backslash X$, then there exist term functions $\sigma, \tau: \mathbf{M}^{n} \rightarrow \mathbf{M}$ on $\underline{\mathbf{M}}$ (that is, morphisms) which agree on $X$ but not at $y$.

PROOF. It is trivial that (a) implies (b), and (b) is equivalent to (c) since (FTC) is precisely the condition for a duality on $\mathscr{A}_{\text {fin }}$ to be a strong duality on $\mathscr{A}_{\text {fin }}$.

Finally, to prove that (c) implies (a), assume (FTC). Let $\mathbf{X}$ be a closed substructure of $\mathbf{M}^{S}$, and let $z \in M^{S} \backslash X$. Then there is a basic clopen set $U$ containing $z$ and not intersecting $X$ given as $U=\left\{x \in M^{S} \mid \pi_{F}(x)=\pi_{F}(z)\right\}$ where $F \subseteq S$ is finite and $\pi_{F}: \mathbf{M}^{S} \rightarrow \mathbf{M}^{F}$ is the projection morphism. Then we have that $\pi_{F}(z) \notin \pi_{F}(X)$ and that $\pi_{F}(\mathbf{X})$ is a closed substructure of $\mathbf{M}^{F}$. By (FTC) there are $F$-ary term functions $\sigma$ and $\tau$ that agree on $\pi_{F}(X)$ but disagree at $\pi_{F}(z)$. Consequently the $S$-ary term functions $\sigma \circ \pi_{F}$ and $\tau \circ \pi_{F}$ agree on $X$ but not at $z$.

The careful reader will note that the image of $\mathbf{X}$ under the morphism $\pi_{F}$ need not be a substructure of $\mathbf{M}^{F}$ if partial operations are present. This point will play a significant role in the remainder of this study. While Lemmas 3.3 and 3.5 highlight the symmetry between $\underline{\mathbf{M}}$ in $\mathscr{A}$ and $\mathbf{M}$ in $\mathscr{X}$ which has characterized our development of duality theory, this symmetry begins to break down in the presence of partial operations in $\mathbf{M}$. For example, partial operations preclude a full converse to Proposition 1.11 of Davey and Werner [16] which we present here. 
3.7 Injectivity Lemma. Assume that $\mathbf{M}$ yields a full duality on $\mathscr{A}$. If $\underline{\mathbf{M}}$ is injective in $\mathscr{A}$, then $\mathbf{M}$ is injective in $\mathscr{X}$. The converse is true when $\mathbf{M}$ is a total structure.

Proof. We will verify the third item of Lemma 3.3. Let $u: \mathbf{A} \rightarrow \mathbf{B}$ in $\mathscr{A}$ and assume that $D(u)$ is an embedding. Because $\mathscr{A}$ is a quasi-variety of (total) algebras, we can factor $u=w \circ v$ where $v: \mathbf{A} \rightarrow \mathbf{C}$ is a surjection and $w: \mathbf{C} \rightarrow \mathbf{B}$ is an embedding. Then $D(u)=D(v) \circ D(w)$. Focusing on $D(w)$ we notice that it is an embedding since $D(u)$ is an embedding, and it is a surjection by Lemma (3.5). Consequently $D(w)$ is an isomorphism, whence $E D(w)$ is an isomorphism as well. By Figure 1, $w=e_{\mathbf{B}}^{-1} \circ E D(w) \circ e_{\mathbf{C}}$ is an isomorphism, and from this it follows that $u$ is a surjection. This establishes (c) of Lemma (3.3) which gives us the injectivity of M.

The converse is established by the same argument applied dually, where we observe that the factoring of $u$ into a surjection followed by an embedding can be mimicked when $\mathbf{M}$ is a total structure.

Combining the Second Duality Theorem (1.6) and the Second Strong Duality Theorem (3.6) we can now give purely finite conditions for the existence of a strong duality when $\mathbf{M}$ is a total structure and $R$ is finite.

3.8 Third Strong Duality Theorem. Assume that $\mathbf{M}=\langle M ; G, R, \mathscr{T}\rangle$ is a total structure and that $R$ is finite. Then the following are equivalent:

(a) $\mathbf{M}$ yields a strong duality on $\mathscr{A}$;

(b) $\mathbf{M}$ yields a strong duality on $\mathscr{A}_{\text {fin }}$;

(c) (IC) and (FTC) hold.

PROOF. (a) implies (b) trivially, so now assume (b). To prove (IC), let $n \in \mathbb{N}$ and let $\mathbf{X} \leq \mathbf{M}^{n}$. By (b) we have that $\mathbf{X}$ is term-closed, and from the finitary version of the Third Duality Theorem (2.4) we conclude that each morphism from $\mathbf{X}$ into $\mathbf{M}$ extends to an $n$-ary term function on $\underline{\mathbf{M}}$. Since (FTC) is given directly by (b), we have (c). Finally, (c) implies (a) since duality follows from (IC) by the Second Duality Theorem (1.6) and strong duality follows from (FTC) by the Second Strong Duality Theorem (3.6).

\section{Producing strong dualities}

In this section we will gather together the theory that we have developed so far and use it to prove two theorems: the Two-for-One Strong Duality Theorem and the 
NU-Strong-Duality Theorem. These two results can be used to produce all presently known full dualities, all of which are, in fact, strong dualities. The Two-for-One Strong Duality Theorem is derived from our description of the dual category $\square D(\mathscr{A})$ as the isomorphs of term-closed sets, while the NU-Strong-Duality Theorem derives from our characterization of $\square D(\mathscr{A})$ as the isomorphs of hom-closed sets. It is here that these two divergent approaches come to fruition.

We first address the case where both partial operations and relations can be avoided so that our strongest results will apply. These results reduce the discussion to properties of $\mathscr{A}_{\text {fin }}$ and $\mathscr{X}_{\text {fin }}$ where topology vanishes. Thus there arises a complete symmetry between the roles of $\underline{\mathbf{M}}$ and $\mathbf{M}$ which allows us to get two strong dualities for the price of one. If $\mathbf{M}=\langle M ; G, \mathscr{T}\rangle$ that is, the sets $H$ of partial operations and $R$ of relations are empty, then we say that $\mathbf{M}$ is a total algebra.

4.1 Two-For-One Strong Duality Theorem. Let $\underline{\mathbf{M}}=\langle M ; F\rangle$ and assume that $\mathbf{M}=\langle M ; G, \mathscr{T}\rangle$ is a total algebra. Define $\underline{\mathbf{M}}^{\prime}=\langle M ; G\rangle$ and $\mathscr{A}^{\prime}=\mathbb{1 S \mathbb { P }} \underline{\mathbf{M}}^{\prime}$, and define $\mathbf{M}^{\prime}=\langle M ; F, \mathscr{T}\rangle$ and $\mathscr{X}^{\prime}=\mathbb{} \mathbb{S}_{c} \mathbb{P} \mathbf{M}^{\prime}$. Then the following are equivalent:

(a) (IC) and (FTC) hold with respect to $\mathbf{M}$;

(b) (IC) and (FTC) hold with respect to $\mathbf{M}^{\prime}$;

(c) the algebras $\underline{\mathbf{M}}$ and $\underline{\mathbf{M}}$ ' satisfy the following symmetric conditions:

(i) every homomorphism from a subalgebra of $\underline{\mathbf{M}}^{n}$ into $\underline{\mathbf{M}}$ extends to an $n$-ary term function on $\underline{\mathbf{M}}^{\prime}$,

(ii) every homomorphism from a subalgebra of $\left(\underline{\mathbf{M}}^{\prime}\right)^{n}$ into $\underline{\mathbf{M}}^{\prime}$ extends to an $n$-ary term function on $\underline{\mathbf{M}}$;

(d) (IC) holds with respect to both $\mathbf{M}$ and $\mathbf{M}^{\prime}$;

(e) $\mathbf{M}$ and $\mathbf{M}^{\prime}$ yield strong dualities on $\mathscr{A}$ and $\mathscr{A}^{\prime}$ respectively.

PROOF. For the purposes of this argument, we will say that $\mathbf{M}$ yields a coduality on $\mathscr{A}\left(\right.$ on $\mathscr{A}_{\text {fin }}$ ) if $\varepsilon_{\mathbf{X}}$ is an isomorphism for each $\mathbf{X}$ in $\mathscr{X}$ (in $\mathscr{X}_{\text {fin }}$ ).

We will first establish the equivalence of (a), (b) and (e). Observe that $\mathbf{M}$ yields a duality (a coduality) on $\mathscr{A}_{\text {fin }}$ if and only if $\mathbf{M}^{\prime}$ yields a coduality (a duality) on $\mathscr{A}_{\text {fin }}^{\prime}$, since exactly the same maps are involved. Consequently, $\mathbf{M}$ yields a full duality on $\mathscr{A}_{\text {fin }}$ if and only if $\mathbf{M}^{\prime}$ yields a full duality on $\mathscr{A}_{\text {fin }}^{\prime}$.

We now claim that the same is true of strong dualities. To prove it, assume that $\mathbf{M}^{\prime}$ yields a strong duality on $\mathscr{A}^{\prime}$. By the First Strong Duality Theorem (3.2), $\mathbf{M}^{\prime}$ is injective in $\mathscr{X}^{\prime}$. Applying the Injectivity Lemma (3.7) we conclude that $\underline{\mathbf{M}}^{\prime}$ is injective in $\mathscr{A}^{\prime}$ and therefore $\mathbf{M}$ is injective in $\mathscr{X}_{\text {fin }}$. Since $\mathbf{M}^{\prime}$ yields a strong duality on $\mathscr{A}_{\text {fin }}^{\prime}$, it also yields a full duality on $\mathscr{A}_{\text {fin }}^{\prime}$ and therefore $\mathbf{M}$ yields a full duality on $\mathscr{A}_{\text {fin }}$. By the First Strong Duality Theorem (3.2) we conclude that $\mathbf{M}$ yields a strong duality on $\mathscr{A}_{\text {fin }}$. From the Third Strong Duality Theorem (3.8) it follows that $M$ yields 
a strong duality on $\mathscr{A}$. Since the converse holds by the same argument, we have that $\mathbf{M}$ yields a strong duality on $\mathscr{A}$ if and only if $\mathbf{M}^{\prime}$ yields a strong duality on $\mathscr{A}^{\prime}$. By the Third Strong Duality Theorem (3.8), the former statement is equivalent to (a) and the latter to (b), and their conjunction is clearly equivalent to (e). Thus (a), (b) and (e) are all equivalent.

Condition (c) is simply an explicit version of (d) expressed as (finitary) properties of the algebras alone. Since (a) and (b) trivially imply (d), it remains to prove the converse: if (IC) holds with respect to both $\mathbf{M}$ and $\mathbf{M}^{\prime}$, then so does (FTC). Again applying the Second Duality Theorem (1.6), the fact that (IC) holds with respect to $\mathbf{M}$ tells us that $\mathbf{M}$ yields a duality on $\mathscr{A}_{\text {fin }}$, that is, $\mathbf{M}^{\prime}$ yields a coduality on $\mathscr{A}_{\text {fin }}^{\prime}$, and, moreover, that $\mathbf{M}$ is injective in $\mathscr{X}_{\text {fin }}$. Similarly, (IC) holding with respect to $\mathbf{M}^{\prime}$ tells us that $\mathbf{M}^{\prime}$ yields a duality on $\mathscr{A}_{\text {fin }}^{\prime}$, that is, $\mathbf{M}$ yields a coduality on $\mathscr{A}_{\text {fin }}$, and, moreover, that $\mathbf{M}^{\prime}$ is injective in $\mathscr{X}_{\text {fin }}^{\prime}$. Putting these facts together and applying the First Strong Duality Theorem (3.2), we conclude that if (d) holds, then $\mathbf{M}$ and $\mathbf{M}^{\prime}$ yield strong dualities on $\mathscr{X}_{\text {fin }}$ and $\mathscr{X}_{\text {fin }}^{\prime}$, respectively. By the Third Strong Duality Theorem (3.8), (FTC) holds with respect to both $\mathbf{M}$ and $\mathbf{M}^{\prime}$, and therefore (a) and (b) hold.

Given $\underline{\mathbf{M}}$, where do we find the algebra $\underline{\mathbf{M}^{\prime}}$ ? Many applications come from the following corollary which tells us when we can use the first candidate that comes to mind.

4.2 Strong SELF-DuALITY THEOREM. Let $\mathbf{M}=\langle M ; F, \mathscr{T}\rangle$ be obtained by augmenting $\underline{\mathbf{M}}=\langle M ; F\rangle$ with the discrete topology. Then $\mathbf{M}$ yields a strong duality on $\mathscr{A}$ (and $\underline{\mathbf{M}}$ is injective in $\mathscr{A}$ ) if and only if (IC) holds.

Underlying our quest for strong dualities is a fundamental question: can every duality be upgraded to a strong duality? We state this question more precisely below.

4.3 Problem. Assume that $\mathbf{M}$ yields a duality on $\mathscr{A}$. Can we extend the structure $\mathbf{M}$ by adding (finitely many) partial or total operations and so obtain a structure $\mathbf{M}^{\prime}$ which yields a strong duality (or a full duality) on $\mathscr{A}$ ?

Consider, for example, a finite algebra $\underline{\mathbf{M}}$ which is a lattice under some pair of binary term functions. The NU-Duality Theorem (1.7) gives us a duality on $\mathscr{A}=\square \mathbb{S P} \underline{\mathrm{M}}$ by taking for $R$ (a generating set of) the binary algebraic relations on $\underline{\mathbf{M}}$. Can finite $G$ and $H$ be chosen to make this into a full duality? a strong duality? We can now give an extension of Theorem 2.37 of Clark and Krauss [5] which provides affirmative answers in all of these cases, producing many new strong dualities. Our approach is to look for partial operations which will insure that substructures of powers of $\mathbf{M}$ are 
hom-closed. The key is to observe that, in a congruence distributive variety, Bjarni Jónsson has already done all but a finite amount of the work for us and that a handful of partial operations will do the rest!

4.4 LEMMA. Let $M$ be a finite set, let $U$ be an ultrafilter on a set $I$, and let $f: M^{I} \rightarrow M$ be defined by

$$
f(\beta)=a \quad \text { if and only if } \quad \beta^{-1}(a) \in U .
$$

Then every topologically closed subspace $X$ of a product space $M^{S}$ is closed under $f$.

Proof. Let $\mathbf{x} \in X^{\prime}$. To show that $\mathbf{f}(\mathbf{x}) \in X$ we let $F \subseteq S$ be finite and check that $\mathbf{f}(\mathbf{x})$ agrees with a member of $X$ on $F$. For each $s \in F$, denote $f\left(\pi_{s} \circ \mathbf{x}\right) \in M$ by $a_{s}$. Thus $\left(\pi_{s} \circ \mathbf{x}\right)^{-1}\left(a_{s}\right) \in U$. Since $F$ is finite,

$$
J=\bigcap\left\{\left(\pi_{s} \circ \mathbf{x}\right)^{-1}\left(a_{s}\right) \mid s \in F\right\} \in U .
$$

Then for any $j \in J$ we find that $\mathbf{f}(\mathbf{x})$ agrees with $\mathbf{x}(j)$ on $F$ since, if $s \in F$, then

$$
\mathbf{f}(\mathbf{x})(s)=f\left(\pi_{s} \circ \mathbf{x}\right)=a_{s}=\left(\pi_{s} \circ \mathbf{x}\right)(j)=\mathbf{x}(j)(s) .
$$

Every congruence on a finite algebra $\mathbf{Q}$ is a meet of meet-irreducible congruences on $\mathbf{Q}$. Let irr $(\mathbf{Q})$ be the least $n$ such that the zero congruence on $\mathbf{Q}$ is a meet of $n$ meet-irreducible congruences, and let

$$
\operatorname{Irr}(\underline{\mathbf{M}})=\max \{\operatorname{irr}(\mathbf{Q}) \mid \mathbf{Q} \text { is a subalgebra of } \underline{\mathbf{M}}\} .
$$

By the clone of $\mathbf{M}$ we mean the clone of partial functions generated by $G \cup H$.

4.5 LEMMA. Assume that $\underline{\mathbf{M}}$ generates a congruence distributive variety and that the clone of $\mathbf{M}$ includes all $n$-ary algebraic partial operations on $\underline{\mathbf{M}}$ for $n \leq \operatorname{Irr}(\underline{\mathbf{M}})$. Then every closed substructure of a power of $\mathbf{M}$ is hom-closed.

PROOF. Let $\mathbf{X} \leq \mathbf{M}^{S}$ be a closed substructure, $\mathbf{B} \leq \underline{\mathbf{M}}^{I}$ a subalgebra and $g$ : $\mathbf{B} \rightarrow \underline{\mathbf{M}}$ a homomorphism. Since $g(\mathbf{B}) \leq \underline{\mathbf{M}}$, there are $k \leq \operatorname{Irr}(\underline{\mathbf{M}})$ meet-irreducible congruences $\psi_{1}, \psi_{2}, \ldots, \psi_{k}$ on $\mathbf{B}$ with $\bigcap \psi_{i}=\operatorname{ker}(g)$. Since $\underline{\mathbf{M}}$ generates a congruence distributive variety we can apply Jónsson's Lemma [20] to get ultrafilters $U_{1}, U_{2}, \ldots, U_{k}$ on $I$ determining congruences $\theta_{1} \subseteq \psi_{1}, \theta_{2} \subseteq \psi_{2}, \ldots, \theta_{k} \subseteq \psi_{k}$ on B. For $i=1,2, \ldots, k$, let $f_{i}: \underline{\mathbf{M}}^{I} \rightarrow \underline{\mathbf{M}}$ be the homomorphism defined by

$$
f_{i}(\beta)=a \quad \text { if and only if } \quad \beta^{-1}(a) \in U_{i}
$$


and let $f=\left(f_{1}, f_{2}, \ldots, f_{k}\right): \underline{\mathbf{M}}^{I} \rightarrow \underline{\mathbf{M}}^{k}$, where $f(\beta)=\left(f_{1}(\beta), f_{2}(\beta), \ldots, f_{k}(\beta)\right)$ for $\beta \in M^{l}$. Let $\mathbf{A} \leq \underline{\mathbf{M}}^{k}$ be the image of $\mathbf{B}$ under $f$, and let $f \uparrow_{B}: \mathbf{B} \rightarrow \mathbf{A}$ be the restriction of $f$. We claim that $g$ can be factored through $f \uparrow_{B}$, that is, that there is a homomorphism $h: \mathbf{A} \rightarrow \underline{\mathbf{M}}$ such that $g=h \circ f \uparrow_{B}$. To prove this we must show that $\operatorname{ker}\left(f \uparrow_{B}\right) \subseteq \operatorname{ker}(g)$. Let $\beta, \gamma \in B$ with $f(\beta)=f(\gamma)$. Then, for $i=1,2, \ldots, k$, we have

$$
f_{i}(\beta)=f_{i}(\gamma)=a_{i} \text { for some } a_{i} \in M .
$$

Consequentiy, $\beta^{-1}\left(a_{i}\right)$ and $\gamma^{-1}\left(a_{i}\right)$ are in $U_{i}$ and therefore $\beta^{-1}\left(a_{i}\right) \cap \gamma^{-1}\left(a_{i}\right) \in U_{i}$. Moreover, $\beta$ and $\gamma$ have the same value, $a_{i}$, on $\beta^{-1}\left(a_{i}\right) \cap \gamma^{-1}\left(a_{i}\right)$ which means that $(\beta, \gamma) \in \theta_{i}$. Since this is true for each $i$, we have

$$
(\beta, \gamma) \in \bigcap\left\{\theta_{i} \mid i=1, \ldots, k\right\} \subseteq \bigcap\left\{\psi_{i} \mid i=1, \ldots, k\right\}=\operatorname{ker}(g) .
$$

From this we conclude that $\operatorname{ker}\left(f \uparrow_{B}\right) \subseteq \operatorname{ker}(g)$.

The structure $\mathbf{X}$ is closed under each $f_{i}$, by Lemma 4.4, and is closed under the $k$-ary partial operation $h$ by hypothesis. Assume that $\mathbf{x} \in X^{I}$ such that, for each $s \in S$, the composition $\pi_{s} \circ \mathbf{X}$ is in $B$. Then

$$
\mathbf{g}(\mathbf{x})(s)=g\left(\pi_{s} \circ \mathbf{x}\right)=h f\left(\pi_{s} \circ \mathbf{x}\right)=h\left(f_{1}\left(\pi_{s} \circ \mathbf{x}\right), f_{2}\left(\pi_{s} \circ \mathbf{x}\right), \ldots, f_{k}\left(\pi_{s} \circ \mathbf{x}\right)\right),
$$

whence $\mathbf{g}(\mathbf{x}) \in X$ since $\mathbf{X}$ is closed under $h$. Thus $\mathbf{X}$ is hom-closed.

This result allows us to offer the following partial solution to Problem 4.3.

4.6 THEOREM. Assume that $\underline{\mathbf{M}}$ generates a congruence distributive variety and that $\mathbf{M}$ yields a duality on $\mathscr{A}$. If $\mathbf{M}^{\prime}$ is obtained from $\mathbf{M}$ by adding to $G \cup H$ all $n$-ary algebraic partial operations where $n \leq \operatorname{Irr}(\underline{\mathbf{M}})$, then $\underline{\mathbf{M}}^{\prime}$ yields a strong duality on $\mathscr{A}$.

Since any algebra which has a near-unanimity term generates a congruence distributive variety (Mitschke [21]), we can combine these results with the NU-Duality Theorem (1.7) to obtain our final strong duality theorem.

4.7 NU-STRONG DUALITY THEOREM. Let $k \geq 2$ and assume that $\underline{\mathbf{M}}$ has $a(k+1)$ ary near-unanimity term. If the structure on $\mathbf{M}$ generates all subalgebras of $\underline{\mathbf{M}}^{k}$ and the clone of $\mathbf{M}$ includes all $n$-ary algebraic partial operations on $\underline{\mathbf{M}}$ for $n \leq \operatorname{Irr}(\underline{\mathbf{M}})$, then $\mathbf{M}$ yields a strong duality on $\mathscr{A}$. 


\section{Examples of strong dualties}

The original presentation of natural dualities of Davey and Werner [16] includes fourteen detailed examples of full dualities which can be obtained in a uniform manner from their methods. We observe here, without examining them individually, that a direct application of our theorems of the previous section will confirm that each of these is indeed a strong duality as well. The strong dualities for vector spaces, abelian groups of exponent at most $n$, and semilattices follow from the Strong Self-Duality Theorem (4.2); the strong duality for sets and the Morita duality for modules require the Two-for-One Strong Duality Theorem (4.1) and all of the remaining examples are obtainable from the NU-Strong Duality Theorem (4.7). For almost all of their examples, Davey and Werner give an axiomatization of the dual category $\mathscr{X}$. Such an axiomatization greatly increases the utility of a full or strong duality. The general question of axiomatizing the topological quasi-variety $\mathscr{X}=\square \mathbb{S}_{c} \mathbb{P} \mathbf{M}$ is considered in depth in Clark and Krauss [5]. For this section we have selected a few new examples of strong dualities that can be established to illustrate further the scope, applicability and limitations of the various theorems we have presented.

Davey, McKenzie and Heindorf [12] have recently shown that, in a congruence distributive variety, the only finite algebras $\underline{\mathbf{M}}$ whose generated quasivariety $\mathscr{A}$ admit a duality at all are those with a near-unanimity term. There are of course many such examples, but they have attracted very little attention partly because it was never known or expected that these dualities could be upgraded to full dualities. Our NUStrong Duality Theorem (4.7) now guarantees that this can be done in every instance. Apart from the Heyting algebras and distributive $p$-algebras given below, we have not pursued this construction in individual cases; particularly not in any cases that would require more than unary (partial) operations. Doing so would mean sufficiently simplifying the structure on $\mathbf{M}$ to make it possible to find a useful description for the dual category $\mathscr{X}$. We hope to see such examples developed and applied in the future.

Heyting Algebras We consider first Heyting algebras $\mathbf{A}=\langle A ; \wedge, \vee, \rightarrow, 0,1\rangle$ consisting of a distributive lattice $\langle A ; \wedge, \vee, 0,1\rangle$ with smallest element 0 and largest element 1, endowed with the induced Heyting implication $\rightarrow$. The linear sum, $\mathbf{A} \oplus \mathbf{B}$, of bounded distributive lattices (Heyting algebras) $\mathbf{A}$ and $\mathbf{B}$ is obtained by placing (the lattice reduct of) A below (that of) B to form a new distributive lattice, and then using this lattice order to define the new Heyting implication $\rightarrow$. The reduced sum of $\mathbf{A}$ and $\mathbf{B}$ is obtained from $\mathbf{A} \oplus \mathbf{B}$ by identifying the 1 of $\mathbf{A}$ with the 0 of $\mathbf{B}$. A finite Heyting algebra $\underline{\mathbf{M}}$ is subdirectly irreducible if and only if has the form $\underline{M} \cong \mathbf{L} \oplus \mathbf{1}$ for some finite distributive lattice $\mathbf{L}$, and in this case all of its subalgebras are subdirectly irreducible as well.

Since $(x \wedge y) \vee(x \wedge z) \vee(y \wedge z)$ determines a majority operation on any Heyting 
algebra, we obtain, from the NU-Strong Duality Theorem (4.7), a strong duality for the quasi-variety generated by $\underline{\mathbf{M}}=\mathbf{L} \oplus \mathbf{1}$ by taking all homomorphisms between subalgebras of $\underline{\mathbf{M}}$ as (partial) operations $H$ on $\underline{\mathbf{M}}$ and all subalgebras of $\underline{\mathbf{M}}^{2}$ as relations $R$ on $\mathbf{M}$. In general this yields a rather unwieldy structure, but there are many cases where it can be reduced to a simple generating set. Davey and Werner [18] consider choices of $\mathbf{L}$ which satisfy the following additional property:

(*) Every subdirectly irreducible subalgebra of a homomorphic image of $\mathbf{L}$ (regarded as a Heyting algebra) can be embedded into $\underline{\mathbf{M}}$.

They show that if $\mathbf{L}$ satisfies (*), then the set Hom (M) of internal homomorphisms of $\underline{\mathbf{M}}$, that is, unary algebraic (partial) operations on $\underline{\mathbf{M}}$, is enough to yield a duality on $\mathscr{A}$. Moreover they prove that there are many such $\mathbf{L}$ by showing that the class of all distributive lattices satisfying $(*)$ is closed under direct product, linear sum and reduced linear sum. As an immediate application of the NU-Strong Duality Theorem (4.7) we see that these dualities are strong and therefore full.

5.1 THEOREM. If $\underline{\mathbf{M}}=\mathbf{L} \oplus \mathbf{1}$ is a finite Heyting algebra such that $\mathbf{L}$ satisfies (*), then $\mathbf{M}=\langle M ; \operatorname{Hom}(\underline{\mathbf{M}}), \mathscr{T}\rangle$ yields a strong duality on $\mathscr{A}=\square \mathbb{S} \mathbb{P} \underline{\mathbf{M}}$.

In more special cases we can use our theory to improve this result by further simplifying the structure on $\mathbf{M}$. We say that $\mathbf{M}$ is (strongly) endo-dualizable if $\mathbf{M}=\langle M$; End $(\mathbf{M}), \mathscr{T}\rangle$ yields a (strong) duality on $\mathscr{A}$. For example, Davey [8] showed that each finite Heyting $n$-chain $\underline{\mathbf{M}}_{n}$ is endo-dualizable. See Davey [10], Davey and Werner [16] and Davey and Priestley [14] for alternative, and progressively simpler, proofs.

5.2 THEOREM. (a) The Heyting algebra $\underline{\mathbf{M}}=2^{2} \oplus 1$ is strongly endo-dualizable.

(b) The Heyting algebra $n$-chain is strongly endo-dualizable if and only if $n<4$.

(c) Let $h$ be the internal isomorphism of the Heyting algebra 4-chain $\underline{\mathbf{M}}_{4}$ between the two three-element subalgebras of $\underline{\mathbf{M}}_{4}$ which moves the third element down to the second. Then the structure $\mathbf{M}_{4}=\left\langle M_{4} ;\right.$ End $\left.\left(\underline{\mathbf{M}}_{4}\right), h, \mathscr{T}\right\rangle$ yields a strong duality on $\mathscr{A}$.

Proof. (a) The simplest proof that $\underline{\mathbf{M}}$ is endo-dualizable is due to Davey and Priestley [14] (see also [11]). It is easy to check that each internal homomorphism of $\underline{\mathbf{M}}$ extends to an endomorphism. The result now follows from Theorem (5.1) and the M-Shift Strong Duality Lemma (3.1).

(b) If $n=2$ or $n=3$, every internal homomorphism extends to an endomorphism and we obtain, as above, strong endo-dualizability. Let $n \geq 4$ and let

$$
M_{n}=\left\{0, a_{1}, \ldots, a_{n-2}, 1\right\} \text { with } 0<a_{1}<\cdots<a_{n-2}<1 .
$$


It is easily seen that $X=M_{n} \backslash\left\{a_{1}\right\}$ is closed under the endomorphisms of $\underline{\mathbf{M}}_{n}$ but is not closed under the partial map $h: X \rightarrow M_{n}$, given by $h\left(a_{2}\right)=a_{1}$ and $h(a)=a$ for $a \neq a_{2}$. Thus $X$ is not hom-closed in $M_{n}$ and consequently $\underline{\mathbf{M}}_{n}$ is not strongly endo-dualizable.

(c) We observe that every internal homomorphism other than $h$ extends to an endomorphism and then argue as in (a).

Continuing in the spirit of item (c), we can find generating sets for the internal homomorphisms of longer finite Heyting algebra chains and then use the M-Shift Strong Duality Lemma (3.1) to simplify their strong dualities.

Distributive $p$-Algebras A distributive p-algebra $\left\langle A ; \vee, \wedge,{ }^{*}, 0,1\right\rangle$ consists of a bounded distributive lattice $\langle A ; \vee, \wedge, 0,1\rangle$ with a pseudocomplementation operation * The proper subvarieties of distributive $p$-algebras form an $\omega$-chain $\mathscr{B}_{0} \subset \mathscr{B}_{1} \subset \mathscr{B}_{2} \subset$ $\ldots$ where $\mathscr{B}_{n}$ is generated by the linear sum $\underline{\mathbf{B}}_{n}=2^{n} \oplus 1$. Stone's theorem [23] shows that $\mathbf{B}_{0}=\langle\{0,1\} ; \mathscr{T}\rangle$ yields a full duality on $\mathscr{B}_{0}$ (the variety of Boolean algebras). Taking $\underline{\mathbf{B}}_{1}=\langle\{0, e, 1\} ; \vee, \wedge, *, 0,1$,$\rangle , where 0<e<1$, Davey $[9,10]$ showed that $\mathbf{B}_{1}=\langle\{0, e, 1\} ; \alpha, \leq, \mathscr{T}\rangle$ yields a duality on $\mathscr{B}_{1}$ (the variety of Stone algebras), where

(i) $\alpha$ fixes 0 and takes $e$ and 1 to 1 , and

(ii) $\leq=\{(0,0),(1,1),(e, e),(1, e)\}$,

and proved this to be a full duality. An alternative proof, more in the style of this paper, was given in Davey and Werner [16]. Later, via a completely different technique, Davey and Werner $[17,18]$ obtained a duality for $\mathscr{B}_{2}$, with generator $\underline{\mathbf{B}}_{2}=\left\langle\{0, p, q, e, 1\} ; \vee, \wedge,{ }^{*}, 0,1\right\rangle$ where $p$ and $q$ are atoms joining to $e$. This duality, which was not previously known to be full, arises by taking

$$
\mathbf{B}_{2}=\langle\{0, p, q, e, 1\} ; \sigma, \alpha, \leqslant, \dashv, \mathscr{T}\rangle,
$$

where

(i) $\sigma$ fixes $0, e$ and 1 , and interchanges $p$ and $q$,

(ii) $\alpha$ takes 0 and $q$ to 0 and takes $1, e$ and $p$ to 1 ,

(iii) $\leqslant$ is the order whose only strict comparability is $1<e$,

(iv) $\dashv=\{(0,0),(1,1),(e, e),(1, e),(0, q),(1, p),(e, p)\}$,

(see Figure 8). For each $n \geq 3$, Davey and Priestley [13] found a duality for $\mathscr{B}_{n}$ via a structure with $n$ binary relations and three total unary operations (endomorphisms of $\left.\underline{\mathbf{B}}_{n}\right)$.

5.3 THEOREM. (a) The above dualities for $\mathscr{B}_{n}$, where $n \leq 2$, are strong dualities .

(b) The above dualities for $\mathscr{B}_{n}$, where $n>2$, are neither strong nor full, but can be made strong by adding (a generating set for) the internal homomorphisms of $\underline{\mathbf{B}}_{n}$. 


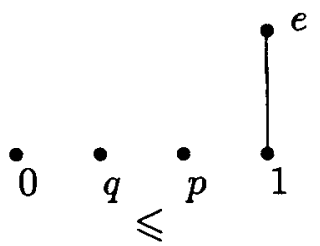

(fully reflexive)

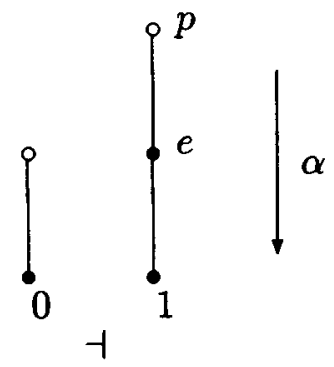

(reflexive at $0.1, e$ )

FIGURE 8. The relations $\leqslant$ and $\dashv$ on $\underline{\mathbf{B}}_{2}$.

PROOF. (a) In each of these cases we apply the NU-Strong Duality Theorem (4.7) to see that a strong duality is obtained by adding to $\mathbf{B}_{n}$ all unary algebraic operations on $\underline{\mathbf{B}}_{n}$. Using the M-Shift Strong Duality Lemma (3.1) we can quickly eliminate all but the given operations.

(b) For $n>2$ we can easily find subsets of $B_{n}$, which are closed under the endomorphisms of $\underline{\mathbf{B}}_{n}$, but are not hom-closed. (For example, let $X$ consist of the top, the bottom and the atoms of $\underline{\mathbf{B}}_{n}$; then $X$ is closed under the endomorphisms of $\underline{\mathbf{B}}_{n}$ but is not closed under the partial unary operations on $\underline{\mathbf{B}}_{n}$.) Since a near-unanimity term is present, (IC) holds and hence, by the Second Duality Theorem (1.6), $\mathbf{B}_{n}$ is injective in $\mathscr{X}$. From the Second Strong Duality Theorem (3.6) we conclude that the duality is not full. Nevertheless the subalgebras of $\underline{\mathbf{B}}_{n}$ are all subdirectly irreducible and hence the NU-Strong Duality Theorem (4.7) assures us that the duality can be made strong by including (a generating set for) for the internal homomorphisms.

Finite Dimensional Vector Spaces over a Finite Field For a finite field $F$, the Strong Self-Duality Theorem (4.2) shows that we obtain a strong duality for the variety $\mathscr{A}$ of vector spaces $\mathbf{A}=\langle A ;+, 0, F\rangle$ over $F$ by taking the one dimensional space $\underline{\mathbf{F}}$ as generator for $\mathscr{A}$ and then choosing $\mathbf{E}$ to be $\underline{\mathbf{F}}$ with the discrete topology. As an application of the Two-For-One Strong Duality Theorem (4.1) that cannot be obtained from the Strong Self-Duality Theorem (4.2), we give another strong duality for $\mathscr{A}$ where we choose $\underline{\mathbf{M}}=\langle M ;+, 0, F\rangle$ to be an arbitrary $n$-dimensional vector space over $F$, where $n>1$. Let $\left\{b_{1}, b_{2}, \ldots, b_{n}\right\}$ be a basis for $\underline{\mathbf{M}}$ and let $\mathbf{B}_{i}$ be the subspace spanned by $b_{i}$. Thus $\underline{\mathbf{M}}=\mathbf{B}_{1} \oplus \mathbf{B}_{2} \oplus \cdots \oplus \mathbf{B}_{n}$ is an internal direct sum. For each $i \leq n$, let $p_{i}: \underline{\mathbf{M}} \rightarrow \mathbf{B}_{i}$ be the $i$ th projection and for $1 \leq i, j \leq n$ let $g_{i j}$ be the unary 
operation on $M$ which interchanges the coefficients of $b_{i}$ and $b_{j}$. Consider the set

$$
G=\{+, 0\} \cup F \cup\left\{g_{i j}\right\} \cup\left\{p_{i}\right\}
$$

of (total) operations on $M$.

5.4 THEOREM. $\mathbf{M}=\langle M ; G, \mathscr{T}\rangle$ yields a strong duality on the (quasi-) variety of vector spaces over $F$ with the arbitrary finite vector space $\underline{\mathbf{M}}=\langle M ;+, 0, F\rangle$ as generating algebra, and $\mathbf{M}^{\prime}=\langle M ;+, 0, F, \mathscr{T}\rangle$ yields a strong duality on the quasi-variety generated by $\underline{\mathbf{M}}^{\prime}=\langle M ; G\rangle$.

We shall prove this theorem by verifying Condition (d) of the Two-for-One Strong Duality Theorem (4.1), namely, that (IC) is satisfied with respect to both $\mathbf{M}$ and $\mathbf{M}^{\prime}$. This will illustrate the kind of interplay that can exist between $\underline{\mathbf{M}}$, which has rather few operations, and $\mathbf{M}$, which has many more. We consider homomorphisms $\tau: \mathbf{X} \rightarrow \mathbf{M}$ and $u: \mathbf{A} \rightarrow \mathbf{M}^{\prime}$ where $\mathbf{X} \leq \mathbf{M}^{m}$ and $\mathbf{A} \leq\left(\mathbf{M}^{\prime}\right)^{m}$. We will see that the many operations on $\mathbf{M}$ insure that $\tau$ has so much to preserve that it can only be (the restriction of) a term function on $\underline{\mathbf{M}}$, and that there are enough operations on $\underline{\mathbf{M}}^{\prime}$ to build $u$ as (the restriction of) a term function on $\underline{\mathbf{M}}^{\prime}$.

\subsection{LEMMA. (IC) holds with respect to $\mathbf{M}$.}

Proof. We first show that $\mathbf{X}$, viewed as a vector space, has a direct sum decomposition as $\mathbf{X}=\mathbf{Y}_{1} \oplus \mathbf{Y}_{2} \oplus \cdots \oplus \mathbf{Y}_{n}$ where each $\mathbf{Y}_{i}$ is a subspace of $\mathbf{B}_{i}^{m}$. Let $\mathbf{Y}_{i}=p_{i} \mathbf{X} \subseteq \mathbf{B}_{i}^{m}$. Then clearly $\mathbf{X} \subseteq \mathbf{Y}_{\mathbf{1}} \oplus \cdots \oplus \mathbf{Y}_{n}$. The reverse containment follows from the fact that $X$ is closed under each $p_{i}$.

Next we show that there are $m$-ary vector space term functions $\tau_{1}, \ldots, \tau_{n}$ such that if $x=y_{1}+\cdots+y_{n}$ according to the above decomposition, then

$$
\tau(x)=\tau_{1}\left(y_{1}\right)+\cdots+\tau_{n}\left(y_{n}\right) .
$$

Let $\tau_{i}$ be the restriction of $\tau$ to $Y_{i}$. For $y \in Y_{i}$ we have

$$
\tau_{i}(y)=\tau(y)=\tau\left(p_{i}(y)\right)=p_{i} \tau(y) \in B_{i}
$$

from which we conclude that $\tau_{i}: \mathbf{Y}_{i} \rightarrow \mathbf{B}_{i}$. Using the duality mentioned above for $\mathbf{B}_{i}$ we conclude that $\tau_{i}$ is an $m$-ary term function.

Finally, we verify that for each $i=1, \ldots, n$ and each $y_{i} \in T_{i}$, we have $\tau_{i}\left(y_{i}\right)=$ $\tau_{1}\left(y_{i}\right)$, from which we will obtain

$$
\begin{aligned}
\tau(x) & =\tau_{1}\left(y_{1}\right)+\cdots+\tau_{n}\left(y_{n}\right) \\
& =\tau_{1}\left(y_{1}\right)+\cdots+\tau_{1}\left(y_{n}\right) \\
& =\tau_{1}\left(y_{1}+\cdots+y_{n}\right)=\tau_{1}(x)
\end{aligned}
$$


for $x=y_{1}+\cdots+y_{n} \in \mathbf{Y}_{1} \oplus \cdots \oplus \mathbf{Y}_{n}$. To do so, let $y_{i}=\left(f_{1} b_{i}, \ldots, f_{m} b_{i}\right)$ where each $f_{j} \in F$. Then

$$
\begin{aligned}
\tau_{i}\left(y_{i}\right) & =\tau\left(y_{i}\right) \\
& =\tau\left(0+\cdots+f_{1} b_{i}+\cdots, \ldots, 0+\cdots+f_{m} b_{i}+\cdots\right) \\
& =\tau g_{1 i}\left(f_{1} b_{1}+\cdots+0+\cdots, \ldots, f_{m} b_{1}+\cdots+0+\cdots\right) \\
& =g_{1 i} \tau\left(f_{1} b_{1}, \ldots, f_{m} b_{1}\right) \\
& =g_{1 i} \tau_{1}\left(f_{1} b_{1}, \ldots, f_{m} b_{1}\right) \\
& =\tau_{1}\left(f_{1} b_{i}, \ldots, f_{m} b_{i}\right)
\end{aligned}
$$

since $\tau_{1}$ is linear, and this is exactly $\tau_{1}\left(y_{i}\right)$.

5.6 LEMMA (IC) holds with respect to $\mathbf{M}^{\prime}$.

Proof. Consider $u: \mathbf{A} \rightarrow \mathbf{M}^{\prime}$ where $\mathbf{A}$ is a subspace of $\left(\mathbf{M}^{\prime}\right)^{m}$. Let $\mathbf{F}$ be the one dimensional space over $F$. If $a \in A$, we can express

$$
u(a)=p_{1} \circ u(a)+\cdots+p_{n} \circ u(a)
$$

Thus we must show, for $i=1, \ldots, n$, that $p_{i} \circ u: \mathbf{A} \rightarrow \mathbf{B}_{i} \leq \mathbf{M}^{\prime}$ is a term function of $\underline{\mathbf{M}}^{\prime}$. For $i=1, \ldots, n$, we can view $\mathbf{A}$ as a subspace of $\mathbf{F}^{m n}$ and $p_{i} \circ u$ as a linear transformation from $\mathbf{A}$ into $\mathbf{B}_{i} \cong \mathbf{F}$. By the duality for $\mathbf{F}$, we know that $p_{i} \circ u$ extends to a term function of $\mathbf{F}$. This means that there is a set

$$
\left\{f_{k j} \mid 1 \leq k \leq m, \quad 1 \leq j \leq n\right\} \subseteq F
$$

such that if $a=\left(a_{1}, \ldots, a_{m}\right) \in A$, where $a_{k}=e_{k 1} b_{1}+\cdots+e_{k n} b_{n} \in M$ and $e_{k j} \in F$, then

$$
p_{i} \circ u(a)=\sum\left\{f_{k j} e_{k j} b_{i} \mid 1 \leq k \leq m, \quad 1 \leq j \leq n\right\} .
$$

Now observe that $e_{k j} b_{i}$ can be obtained from $a_{k}$ by first replacing the coefficient $e_{k i}$ of $b_{i}$ in $a_{k}$ with the coefficient $e_{k j}$ of $b_{j}$ in $a_{k}$, and then extracting the $i$ th coordinate of the result. This gives us

$$
p_{i} \circ u(a)=\sum\left\{f_{k j} p_{i} g_{i j}\left(a_{k}\right) \mid 1 \leq k \leq m, \quad 1 \leq j \leq n\right\},
$$

whence $p_{i} \circ u$ is the restriction of a term function of $\underline{\mathbf{M}}^{\prime}$. 
Finite Abelian Groups The quasi-variety (= variety) $\mathscr{A}$ generated by a finite abelian group $\underline{\mathbf{M}}=\langle M ;+,-, 0\rangle$ is also generated by the finite cyclic group $\underline{\mathbf{M}}_{n}$, of order $n$ for some $n$. If we decide to base our duality for $\mathscr{A}$ on $\underline{\mathbf{M}}_{n}$ rather than on $\underline{\mathbf{M}}$, then after choosing $\mathbf{M}_{n}$ to be $\underline{\mathbf{M}}_{n}$, with the discrete topology, we quickly see that (IC) holds and therefore, by the Strong Self-Duality Theorem (4.2), we find that the duality of Davey and Werner [16] is a strong duality.

Now suppose that we would like to base our duality for $\mathscr{A}$ on the arbitrary finite abelian group $\underline{\mathbf{M}}$ instead. If $\underline{\mathbf{M}}$ happens to have the property that its prime-powerorder cyclic factors are isomorphic for any fixed prime, then we can proceed as we did for vector spaces. Take $\underline{M}^{\prime}$ to be $\underline{\mathbf{M}}$ augmented by the projections onto each of its prime-power-order cyclic factors together with a transposition to interchange each pair of isomorphic coordinates. It is now straight forward to check that the argument given for vector spaces will establish (IC) with respect to both $\mathbf{M}$ and $\mathbf{M}^{\prime}$ and hence, by the Two-for-One Strong Duality Theorem (4.1), we obtain a strong duality for each of the generated quasi-varieties.

If $\underline{\mathbf{M}}$ does not happen to have this special form, then none of the theorems of Section 4 can be used to generate a strong duality. While a strong duality in this case would be unlikely to provide new information about abelian groups, it might very well be a stepping stone to a new wave of strong duality theorems.

\section{References}

[1] M. E. Adams and D. M. Clark, 'Endomorphism monoids in minimal quasi primal varieties', Acta. Sci. Math. (Szeged) 54 (1990), 37-52.

[2] G. Birkhoff, 'On the combination of subalgebras', Math. Proc. Cambridge Phil. Soc. 29 (1933), 441-464.

[3] D. M. Clark, 'Algebraically and existentially closed Stone and double Stone algebras', Symbolic Logic 54 (1989), 363-375.

[4] D. M. Clark and B. A. Davey, 'Natural dualities for the working algebraist', (Cambridge University Press), in preparation.

[5] D. M. Clark and P. H. Krauss, 'Topological quasi varieties', Acta. Sci. Math. (Szeged) 47 (1984), 3-39.

[6] D. M. Clark and J. Schmid, 'The countable homogeneous universal model of $\mathscr{B}_{2}$ ', Studia Logica, to appear.

[7] W. H. Cornish and P. R. Fowler, 'Coproducts of de Morgan algebras', Bull. Austral. Math. Soc. 16 (1977), 1-13.

[8] B. A. Davey, 'Dualities for equational classes of Brouwerian algebras and Heyting algebras', Trans. Amer. Math. Soc. 221 (1976), 119-146.

[9] B. A. Davey, 'Topological dualities for prevarieties of universal algebras', in: Studies in foundations and combinatorics (ed. G.-C. Rota), Adv. Math. Supplementary Studies 1 (Academic Press, Duluth, 1978) pp. 61-99.

[10] B. A. Davey 'Dualities for Stone algebras, double Stone algebras, and relative Stone algebras', Colloq. Math. 46 (1982), 1-14. 
[11] B. A. Davey 'Duality theory on ten dollars a day', in: Algebras and orders (eds. I. G. Rosenberg and G. Sabidussi), NATO Advanced Study Institute Series, Series C 389 (Kluwer, Dordrecht, 1993) pp. 71-111.

[12] B. A. Davey, L. Heindorf and R. McKenzie, 'Near unanimity: an obstacle to general duality theory', Algebra Universalis, to appear.

[13] B. A. Davey and H. A. Priestley 'Optimal natural dualities', Trans. Amer. Math. Soc. 338 (1993), 655-677.

[14] B. A. Davey and H. A. Priestley, 'Optimal dualities for varieties of Heyting algebras', Studia Logica, to appear.

[15] B. A. Davey, R. W. Quackenbush and D. Schweigert, 'Monotone clones and the varieties they determine', Order 7 (1990), 145-168.

[16] B. A. Davey and H. Werner, 'Dualities and equivalences for varieties of algebras', in: Contributions to lattice theory (Szeged, 1980) (eds. A. P. Huhn and E. T. Schmidt) Colloq. Math. Soc. János Bolyai 33 (North-Holland, Amsterdam, 1983) pp. 101-275.

[17] B. A. Davey and H. Werner, 'Piggyback dualities', in: Lectures in universal algebra (Szeged, 1983) (eds. L. Szabó and Á. Szendrei) Coll. Math. Soc. János Bolyai 43 (North-Holland, Amsterdam, 1986) pp. 61-83.

[18] B. A. Davey and H. Werner, 'Piggyback-Dualitäten', Bull. Austral. Math. Soc. 32 (1985), 1-32.

[19] K. H. Hofmann, M. Mislove and A. Stralka, The Pontryagin duality of compact 0-dimensional semilattices and its applications, Lecture Notes in Mathematics Vol. 396 (Springer, Berlin, 1974).

[20] B. Jónsson, 'Algebras whose congruence lattices are distributive', Math. Scand. 21 (1967), 110 121.

[21] A. Mitschke, 'Near unanimity identities and congruence distributivity in equational classes', Algebra Universalis 8 (1978), 29-32.

[22] H. A. Priestley, 'Ordered sets and duality for distributive lattices', Ann. Discrete Math. 23 (1984), 39-60.

[23] M. H. Stone, 'The theory of representations for Boolean algebras', Trans. Amer. Math. Soc. 40 (1936), 37-111.

Mathematics and Computer Science

SUNY, College at New Paltz

NY 12561, USA

Mathematics

La Trobe University

Bundoora

Victoria 3083, Australia 